\title{
Estimating the Location and Time Course of Synaptic Input From Multi-Site Potential Recordings
}

\author{
Steven J. Cox \\ Department of Computational and Applied Mathematics \\ Rice University, 6100 Main St., Houston, TX 77005 \\ cox@rice.edu, Phone: 713-348-5192, Fax: 713-348-5318
}


Abstract: A method is introduced that permits accurate and robust extraction of the location and time course of synaptic conductance from potentials recorded on either side of, and perhaps at some distance from, the synapse in question. It is shown that such data permits one to fully overcome the problems typically associated with lack of spaceclamp. The method does not presume anything about the nature of the time course and yet is applicable to branched, active cells receiving simultaneous input from a number of synapses.

Keywords: Space-clamp, identification, parameter estimation, moment methods, sideways heat equation. 


\section{Introduction}

The objective here is to glean the location and electrical properties of a synapse from the effect it has on potentially distal sites. As synaptic input is small its attenuation at the hands of passive dendritic filtering has been well documented (Rall, 1967; Johnston and Brown, 1983; Rall and Segev, 1985; Koch et al., 1990; Major, 1993; Spruston et al., 1993; Mainen et al., 1996; Bekkers and Stevens, 1996; Jaffe and Carnevale, 1999; Inoue et al., 2001; Hartline and Castelfranco, 2003) yet few (Jack et al., 1971; Pearce, 1993; Häusser and Roth, 1997; Kleppe \& Robinson, 1999) have prescribed remedies. Each of these remedies presumes a particular parametric form for the synaptic time course, considers only somatic potential recordings and confirms the observation (Rall, 1967, pp. 1149) "it is apparent that one cannot infer the location and time course of synaptic input from EPSP shape alone." With the advent of dendritic patch recordings, e.g., (Stuart \& Spruston, 1998; Bernard \& Johnston, 2003) and the promise of quantitative multi-site dye recordings, e.g., (Bullen \& Saggau, 1999; Inoue et al., 2001), it seems natural to investigate the extent to which such data permits one to overcome Rall's observation.

We begin in $\S 2$ with a slight generalization of the problem of (Jack et al., 1971) of determining the location and nature of an $\alpha$-type input current from soma data alone. We produce a concrete example of nonuniqueness and introduce a number of the mathematical methods/obstacles to be used/overcome in subsequent sections. In $\S 3$ we show that potential recordings from both sides of a synapse indeed uniquely and faithfully determines its location and the time course of its conductance. We extend these findings in $\S \S 4$ and 5 to multisynaptic input and to cells with active voltage-gated ion channels. In each instance we test our method on corrupted measurements arising from numerical simulations of model neurons. We determine synaptic locations via the moment method of (Cox \& Griffith, 2001) and recover the respective synaptic time courses by mimicking the Fourier methods of (Eldén et al, 2000).

\section{Recovering a Stereotyped Input Current}

We consider a sealed uniform fiber of length $\ell$ and diameter $d$ with axial resistivity $R_{i}$ and membrane conductance and capacitance $G_{m}$ and $C_{m}$ subject to a synaptic current, $I_{s}(t)$, at $x=x_{s}$. If $V$ and $E_{r}$ denote the local and resting transmembrane potential then 
$V$ obeys the cable equation

$$
\frac{\pi d^{2}}{4 R_{i}} V_{x x}=\pi d\left(C_{m} V_{t}+G_{m}\left(V-E_{r}\right)-I_{s}(t) \delta\left(x-x_{s}\right)\right) \quad 0<x<\ell, \quad 0<t
$$

and the boundary and initial conditions

$$
V_{x}(0, t)=0, \quad V_{x}(\ell, t)=0 \quad \text { and } \quad V(x, 0)=E_{r} .
$$

The $\delta$ function in (2.1) is the standard synaptic footprint, in units of $\mathrm{cm}^{-2}$. On dividing (2.1) by $\pi d G_{m}$ and defining $v \equiv V-E_{r}$ we arrive at

$$
\begin{gathered}
\lambda^{2} v_{x x}=\tau v_{t}+v-V_{s}(t) \delta\left(x-x_{s}\right), \\
v_{x}(0, t)=0, \quad v_{x}(\ell, t)=0 \quad \text { and } \quad v(x, 0)=0
\end{gathered}
$$

where

$$
\lambda^{2}=\frac{d R_{m}}{4 R_{i}}, \quad \tau=R_{m} C_{m}, \quad V_{s}(t)=I_{s}(t) R_{m} \quad \text { and } \quad R_{m}=1 / G_{m}
$$

The objective of Jack et al. (1971) was to suppose that $I_{s}(t)=\alpha^{2} t \exp (-\alpha t)$, and to use knowledge of the soma potential $v(0, t) \equiv v_{0}(t)$ to determine $\alpha$ and $x_{s}$. As a warm-up we investigate the recovery of the slightly more general

$$
I_{s}(t)=\bar{I} \alpha^{2} t \exp (-\alpha t)
$$

For example, for a sealed cable with parameters

$$
\begin{gathered}
d=2 \mathrm{e}-4 \mathrm{~cm}, \quad \ell=0.1 \mathrm{~cm}, \quad C_{m}=1 \mu \mathrm{F} / \mathrm{cm}^{2} \\
G_{m}=0.3 \mathrm{mS} / \mathrm{cm}^{2}, \quad R_{i}=0.15 \mathrm{k} \Omega \mathrm{cm}
\end{gathered}
$$

and whose synaptic location and current is

$$
x_{s}=0.039 \mathrm{~cm} \quad \bar{I}=1 \mu \mathrm{Ams} \mathrm{m}^{-1} \quad \text { and } \quad \alpha=0.5 \mathrm{~ms}^{-1}
$$

we find the response depicted in figure 1.

Jack et al. (1971) argued that the rise time and halfwidth of $v_{0}$ may be used to determine $x_{s}$ and $\alpha$. In reviewing their work Tuckwell (1988) incorrectly argued that the soma potential uniquely determines $x_{s}$ and $\alpha$ and went on to advocate the use of nonlinear least squares and the Laplace transform in their recovery. Regarding uniqueness, he stated that as each pair, $\left(x_{s}, \alpha\right)$, yields a unique soma potential said potential must uniquely determine the pair, $\left(x_{s}, \alpha\right)$. By this reasoning we may conclude that as each real number 
has a unique square, each square must have a unique square root! We shall see below that the first three moments of the soma potential do not uniquely determine the three synaptic parameters. We do this by following Tuckwell's advice and invoke the Laplace transform. We denote this transform by

$$
\hat{v}(x, \xi) \equiv \int_{0}^{\infty} v(x, t) \exp (-\xi t) d t \quad \text { and } \quad \hat{V}_{s}(\xi) \equiv \int_{0}^{\infty} V_{s}(t) \exp (-\xi t) d t
$$

and so transform (2.3) to the ordinary differential equation

$$
\begin{array}{r}
\lambda^{2} \hat{v}_{x x}(x, \xi)=(1+\tau \xi) \hat{v}(x, \xi)-\hat{V}_{s}(\xi) \delta\left(x-x_{s}\right) \\
\hat{v}_{x}(0, \xi)=0, \quad \hat{v}_{x}(\ell, \xi)=0 \quad \text { and } \quad \hat{v}(0, \xi)=\hat{v}_{0}(\xi)
\end{array}
$$

With $\mu(\xi) \equiv \sqrt{1+\tau \xi} / \lambda$ we note that $\hat{v}$ is simply

$$
\hat{v}(x, \xi)= \begin{cases}\hat{v}_{0}(\xi) \cosh (\mu(\xi) x) & \text { if } 0 \leq x \leq x_{s} \\ \hat{v}(\ell, \xi) \cosh (\mu(\xi)(\ell-x)) & \text { if } x_{s} \leq x \leq \ell\end{cases}
$$

This representation is circular in the sense that $\hat{v}(\ell, \xi)$ appears on each side. We remedy this by matching the representations at $x_{s}$. Namely

$$
\hat{v}_{0}(\xi) \cosh \left(\mu(\xi) x_{s}\right)=\hat{v}(\ell, \xi) \cosh \left(\mu(\xi) y_{s}\right)
$$

where $y_{s} \equiv \ell-x_{s}$, implies that

$$
\hat{v}(\ell, \xi)=\hat{v}_{0}(\xi) \frac{\cosh \left(\mu(\xi) x_{s}\right)}{\cosh \left(\mu(\xi) y_{s}\right)}
$$

Our next step is to note that integration of the transformed cable equation, $(2.7)$, in $x$ over the small interval $\left(x_{s}-\varepsilon, x_{s}+\varepsilon\right)$ produces

$$
\lambda^{2}\left(\hat{v}_{x}\left(x_{s}+\varepsilon, \xi\right)-\hat{v}_{x}\left(x_{s}-\varepsilon, \xi\right)\right)=(1+\tau \xi) \int_{x_{s}-\varepsilon}^{x_{s}+\varepsilon} \hat{v}(x, \xi) d x-\hat{V}_{s}(\xi)
$$

As $\hat{v}$ is bounded its integral vanishes in the limit. More precisely, as $\varepsilon \rightarrow 0(2.10)$ takes the form

$$
\lambda^{2}\left(\hat{v}_{x}\left(x_{s}^{+}, \xi\right)-\hat{v}_{x}\left(x_{s}^{-}, \xi\right)\right)=-\hat{V}_{s}(\xi)
$$

If we now substitute (2.8) and (2.9) into (2.11) we find

$$
\lambda^{2} \hat{v}_{0}(\xi) \mu(\xi)\left\{\cosh \left(\mu(\xi) x_{s}\right) \tanh \left(\mu(\xi) y_{s}\right)+\sinh \left(\mu(\xi) x_{s}\right)\right\}=\hat{V}_{s}(\xi)
$$


or, after a little simplification and evaluation of $\hat{V}_{s}$,

$$
\lambda^{2} \hat{v}_{0}(\xi) \mu(\xi) \sinh (\mu(\xi) \ell)=\frac{\bar{I} R_{m} \alpha^{2}}{(\xi+\alpha)^{2}} \cosh \left(\mu(\xi) y_{s}\right)
$$

This is a functional relation between the unknowns $x_{s}, \bar{I}$ and $\alpha$, and so it ought to suffice to consider three instantiations of (2.13). Of the many possibilities we choose, following (Cox \& Griffith, 2001), to evaluate (2.13) and its first two derivatives in $\xi$ at $\xi=0$. This has the advantage of permitting us to knock off $\bar{I}$ and then $\alpha$ and so arrive at a single equation for $x_{s}$ or, equivalently, $y_{s}$. We recall the following connection between such derivatives and the low order moments,

$$
\hat{v}_{0}(0)=\int_{0}^{\infty} v_{0}(t) d t, \quad \hat{v}_{0}^{\prime}(0)=-\int_{0}^{\infty} t v_{0}(t) d t \quad \text { and } \quad \hat{v}_{0}^{\prime \prime}(0)=\int_{0}^{\infty} t^{2} v_{0}(t) d t .
$$

Setting $\xi=0$ in $(2.13)$ yields $\lambda \hat{v}_{0}(0) \sinh (\ell / \lambda)=\bar{I} R_{m} \cosh \left(y_{s} / \lambda\right)$ and so we may solve for the synaptic weight in terms of the synaptic site, i.e.,

$$
\bar{I}=\frac{\lambda G_{m} \hat{v}_{0}(0) \sinh (\ell / \lambda)}{\cosh \left(y_{s} / \lambda\right)}
$$

Next we differentiate each side of (2.13) with respect to $\xi$ and set $\xi=0$ and find

$\lambda\left(2 \hat{v}_{0}^{\prime}(0)+\tau \hat{v}_{0}(0)\right) \sinh (\ell / \lambda)+\hat{v}_{0}(0) \tau \ell \cosh (\ell / \lambda)=\bar{I} R_{m}\left\{\tau y_{s} \sinh \left(y_{s} / \lambda\right) / \lambda-4 \cosh \left(y_{s} / \lambda\right) / \alpha\right\}$

On substituting (2.14) we find an expression of the rate in terms of the site

$$
\alpha=\frac{-4 \lambda \hat{v}_{0}(0)}{\lambda\left(2 \hat{v}_{0}^{\prime}(0)+\tau \hat{v}_{0}(0)\right)+\hat{v}_{0}(0) \tau \ell \operatorname{coth}(\ell / \lambda)-\hat{v}_{0}(0) \tau y_{s} \tanh \left(y_{s} / \lambda\right)}
$$

Finally we equate second derivatives

$$
\begin{aligned}
& \lambda \hat{v}_{0}^{\prime \prime}(0) \sinh (\ell / \lambda)+\lambda \tau \hat{v}_{0}^{\prime}(0)(\sinh (\ell / \lambda)+(\ell / \lambda) \cosh (\ell / \lambda)) \\
&+\left(\tau^{2} / 4\right) \hat{v}_{0}(0)\left(\left(\ell^{2} / \lambda-\lambda\right) \sinh (\ell / \lambda)+\ell \cosh (\ell / \lambda)\right) \\
&=\bar{I} R_{m}\left\{\left(\frac{y_{s}^{2} \tau^{2}}{4 \lambda^{2}}+\frac{6}{\alpha^{2}}\right) \cosh \left(y_{s} / \lambda\right)-\frac{y_{s} \tau}{\lambda}\left(\frac{\tau}{4}+\frac{2}{\alpha}\right) \sinh \left(y_{s} / \lambda\right)\right\}
\end{aligned}
$$

The left hand side is a constant, say $c$, that depends on the data and passive cell properties while the right hand side, after substitution for $\bar{I}$ and $\alpha$ from (2.14) and (2.15), is an explicit function, let us call it $F$, of the synaptic site, $y_{s}$. In other words, (2.16), is merely

$$
c=F\left(y_{s}\right) \text {. }
$$


Of course (2.17) uniquely determines $y_{s}$ so long as $F$ is monotone. We show in figure 2 that $F$ is not, in general, monotone.

We see in fig. 2(A) that the data does indeed uniquely determine the true site, $\ell-x_{s}=0.061$, but that in $2(\mathrm{~B})$, in attempting to recover a faster synapse, the data gives us two choices. The first crossing corresponds to the true one while the second gives a much closer $x_{s}$ and a weaker $\bar{I}$ and a slower $\alpha$. This 'false' synapse nonetheless produces a soma response, see fig. 2(C), that has the same rise and decay characteristics as the true response. We note that all we have shown is that the first three terms in the Maclaurin expansion of $\xi \mapsto \hat{v}_{0}(\xi)$ do not uniquely determine the three synaptic parameters. This is not to say that no three functionals of $\xi \mapsto \hat{v}_{0}(\xi)$ will suffice. In fact, fitting the measured $\hat{v}_{0}(\xi)$ to the predicted $\hat{v}_{0}(\xi)$ over the interval $\xi \in[0.1,1]$ via the Matlab function 1sqcurvefit in the fast $(\alpha=1.5)$ context returned the correct synaptic parameters starting from a variety of different guesses.

A similar strategy is invoked by (Kleppe \& Robinson, 1999) in a two stage process where they fit the soma currents associated with an NMDA current (modeled as a step) and an AMPA current (modeled as a difference of exponentials). More precisely, they first read the location, $x_{s}$, and magnitude of the NMDA component, $I_{N M D A}$ by fitting the measured soma current to that predicted by solving the voltage clamped semi-inifinite cable

$$
\begin{aligned}
& \lambda^{2} v_{x x}=\tau v_{t}+v-I_{N M D A} R_{m} \delta\left(x-x_{s}\right), \\
& v(0, t)=0 \quad \text { and } \quad v(x, 0)=0, \quad 0<x<\infty
\end{aligned}
$$

They then proceed to identify the AMPA current amplitude, $I_{A M P A}$, and time constants $k_{1}$ and $k_{2}$ by fitting the measured soma current to that predicted by

$$
\begin{aligned}
& \lambda^{2} v_{x x}=\tau v_{t}+v-I_{A M P A}\left(\exp \left(-k_{2} t\right)-\exp \left(-k_{1} t\right)\right) R_{m} \delta\left(x-x_{s}\right), \\
& v(0, t)=0 \quad \text { and } \quad v(x, 0)=0, \quad 0<x<\infty
\end{aligned}
$$

They offer empirical evidence that the fits are good, but do not show that the data uniquely constrains each of the synaptic parameters.

We argue in the sections to follow that knowledge of the potential on both sides of a synapse removes this ambiguity. In fact it permits us to uniquely recover synaptic conductance changes of arbitrary shape.

\section{Recovering a General Conductance Change}

Rather than direct current injection we now suppose our uniform fiber experiences a 
conductance change, $g$, and a driving force associated with a synaptic reversal potential, $E$, relative to rest. More precisely, we replace (2.3) with

$$
\lambda^{2} v_{x x}=\tau v_{t}+v+g(t) \delta\left(x-x_{s}\right)(v-E) .
$$

(Häusser \& Roth, 1997) follow (Pearce, 1993) and propose a voltage jump method for recovering a $g$ of the form

$$
g(t)=\bar{g}_{1}\left(\exp \left(-t / d_{1}\right)-\exp (-t / r)\right)+g_{2}\left(\exp \left(-t / d_{2}\right)-\exp (-t / r)\right)
$$

by fitting the soma current, associated with the one parameter family of somatic voltage jumps

$$
v(0, t ; \sigma)= \begin{cases}E_{1} & \text { if } t<\sigma \\ E_{2} & \text { if } t>\sigma\end{cases}
$$

to an approximate predicted parametric form. They argue, based on simulations, that this fit permits accurate determination of the decay rate(s) in (3.2). This method requires measurement of the time course of the somatic current at six or more values of $\sigma$. We wish here to replace this requirement with the less stringent one of simultaneously measuring the membrane potential on either side of the synapse. To begin, we presume sealed ends and a cell initially at rest and that we have measured the end potentials, i.e.,

$$
v(0, t) \equiv v_{0}(t) \quad \text { and } \quad v(\ell, t) \equiv v_{1}(t)
$$

associated with a single synaptic event at $x_{s}$. For example, exciting the cell described by (2.5) with a conductance at and of the form

$$
x_{s}=0.039 \mathrm{~cm} \quad g(t)=(5 \mathrm{e}-3) t^{4} \exp (-t) \quad E=60 \mathrm{mV}
$$

produces the response seen in fig. 3. If the left side of (3.10) lies outside the range of the right side then this indicates that the fiber received input from more than one synapse, a possibility we shall postpone considering until the next section.

We now show how the data in figure 3(B) uniquely determines both $x_{s}$ and $g$. Unlike the previous section, the product $g(t) v(x, t)$ in (3.1) prohibits us from taking an immediate Laplace transform.

We begin by noting that $v$ satisfies the unforced partial differential equation

$$
\lambda^{2} v_{x x}=\tau v_{t}+v
$$


on either side of $x_{s}$. If we presume, naturally, that $v$ starts from and eventually returns to rest then the zeroth order moment

$$
v^{0}(x) \equiv \int_{0}^{\infty} v(x, t) d t
$$

satisfies the unforced ordinary differential

$$
\lambda^{2}\left(v^{0}\right)^{\prime \prime}(x)=v^{0}(x)
$$

on either side of $x_{s}$. Denoting by $v_{0}^{0}$ and $v_{1}^{0}$ the zeroth order moments of our two end potentials and by $v_{l}^{0}$ and $v_{r}^{0}$ the solutions of (3.7) to the left and right of $x_{s}$ respectively, we note that $v_{l}^{0}$ obeys the initial conditions $v_{l}^{0}(0)=v_{0}^{0}$ and $\left(v_{l}^{0}\right)^{\prime}(0)=0$ and so

$$
v_{l}^{0}(x)=v_{0}^{0} \cosh (x / \lambda)
$$

while $v_{r}^{0}$ obeys the terminal conditions $v_{r}^{0}(\ell)=v_{1}^{0}$ and $\left(v_{r}^{0}\right)^{\prime}(\ell)=0$ and so

$$
v_{r}^{0}(x)=v_{1}^{0} \cosh ((x-\ell) / \lambda)
$$

As the potential is continuous across the synapse we may equate $v_{r}^{0}\left(x_{s}\right)$ and $v_{r}^{0}\left(x_{s}\right)$ and find

$$
\frac{v_{0}^{0}}{v_{1}^{0}}=\frac{\cosh \left(\left(x_{s}-\ell\right) / \lambda\right)}{\cosh \left(x_{s} / \lambda\right)}
$$

The left hand side is a simple ratio of means of computed data while the right hand side is an explicit strictly decreasing function of the unknown $x_{s}$ and so (3.10) uniquely determines the synaptic site, $x_{s}$. See figure $4(\mathrm{~A})$ for its application to the cell and synapse used in figure 3 .

We now proceed to recover the time course $g$ at $x_{s}$. We start with a calculation very much like that that brought us (2.11). Namely, integration of (3.1) over a vanishingly small interval about $x_{s}$ brings the representation

$$
g(t)=\lambda^{2} \frac{v_{x}\left(x_{s}^{+}, t\right)-v_{x}\left(x_{s}^{-}, t\right)}{v\left(x_{s}, t\right)-E}
$$

of the unknown $g$. We now show how to recover the synaptic potential $v\left(x_{s}, t\right)$ and the associated axial currents $v_{x}\left(x_{s}^{+}, t\right)$ and $v_{x}\left(x_{s}^{-}, t\right)$ from knowledge of $x_{s}$ and the two end potentials. 
Starting from the left we note that $v$ satisfies

$$
\begin{aligned}
& \lambda^{2} v_{x x}=\tau v_{t}+v \quad 0<x<x_{s} \\
& v(0, t)=v_{0}(t), \quad v_{x}(0, t)=0
\end{aligned}
$$

This is a variant of the well-studied sideways heat equation. The adjective preceding heat stems from the fact that one typically specifies one initial condition and one boundary condition and solves the heat equation forward in time. In our case we have two boundary conditions and wish to solve it sideways in space up to the point $x=x_{s}$. We follow Elden et al. (2000) and accomplish this via the Fourier Transform in time. We denote this transform by

$$
\hat{v}(x, \omega)=\int_{0}^{\infty} v(x, t) \exp (-i t \omega) d t,
$$

and proceed to transform the (proximal) cable equation (3.5) to the ordinary differential equation

$$
\begin{aligned}
\lambda^{2} \hat{v}_{x x}(x, \omega) & =(i \tau \omega+1) \hat{v}(x, \omega) \quad 0<x<x_{s} \\
\hat{v}(0, \omega) & =\hat{v}_{0}(\omega), \quad \hat{v}_{x}(0, \omega)=0 .
\end{aligned}
$$

We set $\mu(\omega) \equiv \sqrt{1+i \tau \omega} / \lambda$ and find

$$
\hat{v}(x, \omega)=\hat{v}_{0}(\omega) \cosh (x \mu(\omega))
$$

and so $v(x, t)$ is simply the inverse Fourier transform of (3.13). As high frequency noise in $v_{0}$ may undermine this transform we introduce a cutoff frequency, $\omega_{c}$, in our representation

$$
\begin{aligned}
v\left(x_{s}, t\right) & =\frac{1}{2 \pi} \int_{-\omega_{c}}^{\omega_{c}} \hat{v}_{0}(\omega) \cosh \left(x_{s} \mu(\omega)\right) \exp (i t \omega) d \omega \\
v_{x}\left(x_{s}^{-}, t\right) & =\frac{1}{2 \pi} \int_{-\omega_{c}}^{\omega_{c}} \hat{v}_{0}(\omega) \mu(\omega) \sinh \left(x_{s} \mu(\omega)\right) \exp (i t \omega) d \omega
\end{aligned}
$$

The choice of $\omega_{c}$ is dictated by examination of the frequency spectrum of $v_{0}$. In fig. $4(\mathrm{~B})$ we have plotted the magnitude of the Fourier transform of the $v_{0}$ depicted in fig. 3(B).

In a similar fashion the transformed (distal) cable equation reads

$$
\begin{aligned}
\lambda^{2} \hat{v}_{x x}(x, \omega) & =(i \tau \omega+1) \hat{v}(x, \omega) \quad x_{s}<x<\ell \\
\hat{v}(\ell, \omega) & =\hat{v}_{1}(\omega), \quad \hat{v}_{x}(\ell, \omega)=0
\end{aligned}
$$

and so,

$$
\hat{v}(x, \omega)=\hat{v}_{1}(\omega) \cosh ((\ell-x) \mu(\omega)),
$$


from which we deduce (again making use of $y_{s}=\ell-x_{s}$ ) that

$$
\begin{aligned}
v\left(x_{s}, t\right) & =\frac{1}{2 \pi} \int_{-\omega_{c}}^{\omega_{c}} \hat{v}_{1}(\omega) \cosh \left(y_{s} \mu(\omega)\right) \exp (i t \omega) d \omega \\
v_{x}\left(x_{s}^{+}, t\right) & =\frac{-1}{2 \pi} \int_{-\omega_{c}}^{\omega_{c}} \hat{v}_{1}(\omega) \mu(\omega) \sinh \left(y_{s} \mu(\omega)\right) \exp (i t \omega) d \omega
\end{aligned}
$$

If $E$ is presumed known then (3.14) and (3.15) complete the determination (see fig. 4) of $g$ via (3.11). Our implementation of (3.14) and (3.15) uses the fast Fourier transform in much the same manner as the lovely shetools Matlab package at http://www.mai.liu.se/ frber/heat_equation/sof tware.html.

This result demonstrates that our method accurately recovers both $x_{s}$ and $g$ from noisy end potentials. We now address a number of generalizations of our method.

(1) Somatic Shunt: If the fiber arises from a soma then the sealed end condition, $v_{x}(0, t)=0$, is replaced with

$$
\frac{\pi d^{2}}{4 R_{i}} v_{x}(0, t)=A_{s}\left(C_{s} v_{t}(0, t)+G_{s} v(0, t)\right)
$$

where $A_{s}$ is the surface area of the soma while $C_{s}$ and $G_{s}$ denote the soma membrane capacitance and conductance respectively. It follows that the associated $v^{0}$ obeys

$$
\left(v^{0}\right)^{\prime}(0)=\beta v^{0}, \quad \text { where } \beta=\frac{4 A_{s} R_{i} G_{s}}{\pi d^{2}}
$$

and, as such, the $v_{l}^{0}$ of (3.8) becomes $v_{l}^{0}(x)=v_{0}^{0}\{\cosh (x / \lambda)+\beta \lambda \sinh (x / \lambda)\}$ and so the analog of $(3.10)$ is

$$
\frac{v_{0}^{0}}{v_{1}^{0}}=\frac{\cosh \left(\left(x_{s}-\ell\right) / \lambda\right)}{\cosh \left(x_{s} / \lambda\right)+\beta \lambda \sinh \left(x_{s} / \lambda\right)} .
$$

As the right hand side is a strictly decreasing function of $x_{s}$ it follows as above that the ratio of the time integrals of the two end potentials uniquely determines the site of the synapse. Regarding the synaptic time course, all proceeds as before except for the simple modification

$$
\hat{v}(x, \omega)=\hat{v}_{0}(\omega)\left\{\cosh (x \mu(\omega))+\left\{\left(\beta+4 R_{i} C_{s} A_{s} i \omega\right) / \mu(\omega)\right\} \sinh (x \mu(\omega))\right\}
$$

to $(3.13)$.

(2) Nonterminal measurement sites: If the potential is measured at $x_{0}$ and $x_{1}$ where $0<x_{0}<x_{s}<x_{1}<\ell$ we may argue as above once we compute $v_{x}(x, t)$ at $x_{0}$ and 
$x_{1}$. To accomplish this first solve the well-posed cable problems on $\left[0, x_{0}\right]$ and $\left[x_{1}, \ell\right]$ respectively

$$
\begin{array}{llrl}
\lambda^{2} v_{x x}=\tau v_{t}+v, & v_{x}(0, t)=0 & v\left(x_{0}, t\right)=v_{0}(t) \\
\lambda^{2} v_{x x}=\tau v_{t}+v, & v\left(x_{1}, t\right)=v_{1}(t) & v_{x}(\ell, t)=0
\end{array}
$$

and then simply evaluate $v_{x}(x, t)$ at $x_{0}$ and $x_{1}$. With $v$ and $v_{x}$ at both $x_{0}$ and $x_{1}$ we can now solve inward from both sides toward $x_{s}$. In this case, the $x_{s}$ on the right in (3.14) will be replaced with $x_{s}-x_{0}$ and the $y_{s}$ in (3.15) with $x_{1}-x_{s}$. As sinh and cosh are both monotonic we note that the closer $x_{0}$ and $x_{1}$ are to $x_{s}$ the smaller will be the factor by which the transformed data, $\hat{v}_{0}$ and $\hat{v}_{1}$, is multiplied. In other words, the error in the recovery decreases as the distance from the recording sites to the synaptic site decreases.

(3) Branched Cell: Consider the fork in figure 5. We have numbered its branches 1,2 and 3. We suppose synaptic input at one site chosen from $\mathrm{a}, \mathrm{b}$ and $\mathrm{c}$ and that we have measured the potential at the 3 sealed sites $\mathrm{A}, \mathrm{B}$ and $\mathrm{C}$. We have denoted the branchpoint by $\lambda$. We first determine the branch receiving synaptic input by solving sideways on each branch from the terminal in and up to $\lambda$. If the potentials computed in this way along branches 1 and 2 happen to agree at $\lambda$ then we may conclude that the synapse lies on branch 3. As the computations along branch 1 and 2 also yield their current into $\lambda$ we may deduce, at $\lambda$, the potential and its spatial derivative along branch 3. From there we may apply our existing approach to the recover of the location and time course of the synapse at c. This line of reasoning permits us to establish

Proposition 1. The location and time course of monosynaptic input into an arbitrarily branched, sealed, passive cell is uniquely and constructively determined by measurement of the potential at each of the terminals.

(4) Unknown $E$ : If the synaptic reversal potential is unknown then one may remove it from (early) consideration by pairing synaptic input with two distinct somatic current injections. More precisely, we may denote by $v^{(k)}$, where $k=1$ or 2 , the solution to

$$
\lambda^{2} v_{x x}=\tau v_{t}+v+g(t) \delta\left(x-x_{s}\right)(v-E), \quad v_{x}(0, t)=i_{0}^{(k)}(t) \quad v_{x}(\ell, t)=0
$$

and note that $v \equiv v^{(1)}-v^{(2)}$ satisfies

$$
\lambda^{2} v_{x x}=\tau v_{t}+v+g(t) \delta\left(x-x_{s}\right) v, \quad v_{x}(0, t)=i_{0}(t) \quad v_{x}(\ell, t)=0
$$


where $i_{0} \equiv i_{0}^{(1)}-i_{0}^{(2)}$. This equation for $v$ is independent of $E$ and so we may unambiguously solve for

$$
g(t)=\lambda^{2} \frac{v_{x}\left(x_{s}^{+}, t\right)-v_{x}\left(x_{s}^{-}, t\right)}{v\left(x_{s}, t\right)}
$$

where each of the 3 functions on the right follow, as above, from propagating inward the Fourier transforms of the end data. Though vanishing end currents kept our formulae clean they are not a precondition of our method. Once $g$ is known one can finally read off $E$ from (3.11).

Generalization of this method to the multisynaptic case requires a bit more care and so we embark on a new section.

\section{Multisynaptic Input}

We return to the uniform sealed fiber but permit simultaneous synaptic input at sites $\left\{x_{s_{1}}, \ldots, x_{s_{N}}\right\}$ with time courses $\left\{g_{1}, \ldots, g_{N}\right\}$ driven by reversal potentials $\left\{E_{1}, \ldots, E_{N}\right\}$. The cable equation now reads

$$
\lambda^{2} v_{x x}=\tau v_{t}+v+\sum_{n=1}^{N} g_{n}(t) \delta\left(x-x_{s_{n}}\right)\left(v-E_{n}\right)
$$

We begin with $N=2$ and suppose we have measured the potential at $\left\{0, x_{1}, \ell\right\}$ where

$$
0<x_{s_{1}}<x_{1}<x_{s_{2}}<\ell
$$

The rub here is that we do not know $v_{x}$ at $x_{1}$. Hence, the simple matching of means at $x_{s_{1}}$ and $x_{s_{2}}$ will not uniquely determine them. We augment these means therefore with first order moments. More precisely, we introduce

$$
v^{1}(x) \equiv \int_{0}^{\infty} t v(x, t) d t
$$

and note that

$$
\int_{0}^{\infty} t v_{t}(x, t) d t=-\int_{0}^{\infty} v(x, t) d t=-v^{0}(x)
$$

and so multiplying (4.1) by $t$ and integrating in time gives

$$
\lambda^{2} v_{x x}^{1}=v^{1}-\tau v^{0}
$$


away from the synaptic sites. We shall now solve for $v^{0}$ and $v^{1}$ in the left, middle and right intervals, i.e., in $\left[0, x_{s_{1}}\right],\left[x_{s_{1}}, x_{s_{2}}\right]$ and $\left[x_{s_{2}}, \ell\right]$. The solutions in the middle interval will depend on the two new unknowns

$$
a \equiv \int_{0}^{\infty} v_{x}\left(x_{1}, t\right) d t \quad \text { and } \quad b \equiv \int_{0}^{\infty} t v_{x}\left(x_{1}, t\right) d t
$$

We shall see however that matching the $v^{0}$ and the $v^{1}$ at $x_{s_{1}}$ and $x_{s_{2}}$ give us 4 equations in the 4 unknowns, $\left\{x_{s_{1}}, x_{s_{2}}, a, b\right\}$. As $a$ and $b$ appear linearly we represent them in terms of the synaptic sites and so must solve two nonlinear equations for the two unknown synaptic sites.

We first state and solve the six sub-problems. The solution of the zero order left problem,

$$
\lambda^{2} v_{x x}^{0}=v^{0}, \quad v^{0}(0)=v_{0}^{0}, \quad v_{x}^{0}(0)=0, \quad 0<x<x_{s_{1}}
$$

is

$$
v_{l}^{0}(x)=v_{0}^{0} \cosh (x / \lambda) .
$$

The solution of the first order left problem,

$$
\lambda^{2} v_{x x}^{1}=v^{1}-\tau v_{0}^{0} \cosh (x / \lambda), \quad v^{1}(0)=v_{0}^{1}, \quad v_{x}^{1}(0)=0, \quad 0<x<x_{s_{1}}
$$

is

$$
v_{l}^{1}(x)=v_{0}^{1} \cosh (x / \lambda)-\tau v_{0}^{0} x \sinh (x / \lambda) /(2 \lambda)
$$

The solution of the zero order middle problem,

$$
\lambda^{2} v_{x x}^{0}=v^{0}, \quad v^{0}\left(x_{1}\right)=v_{1}^{0}, \quad v_{x}^{0}\left(x_{1}\right)=a . \quad x_{s_{1}}<x<x_{s_{2}}
$$

is

$$
v_{m}^{0}(x)=v_{1}^{0} \cosh \left(\left(x_{1}-x\right) / \lambda\right)+a \lambda \sinh \left(\left(x-x_{1}\right) / \lambda\right)
$$

The solution of the first order middle problem,

$$
\begin{gathered}
\lambda^{2} v_{x x}^{1}=v^{1}-\tau\left(v_{1}^{0} \cosh \left(\left(x_{1}-x\right) / \lambda\right)+a \lambda \sinh \left(\left(x-x_{1}\right) / \lambda\right)\right), \quad x_{s_{1}}<x<x_{s_{2}} \\
v^{1}\left(x_{1}\right)=v_{1}^{1}, \quad v_{x}^{1}\left(x_{1}\right)=b
\end{gathered}
$$

is

$v_{m}^{1}(x)=\frac{2 v_{1}^{1}+\tau a\left(x_{1}-x\right)}{2} \cosh \left(\left(x-x_{1}\right) / \lambda\right)+\frac{\lambda^{2}(2 b+\tau a)+\tau v_{1}^{0}\left(x_{1}-x\right)}{2 \lambda} \sinh \left(\left(x-x_{1}\right) / \lambda\right)$ 
The solution of the zero order right problem,

$$
\lambda^{2} v_{x x}^{0}=v^{0}, \quad v^{0}(\ell)=v_{2}^{0}, \quad v_{x}^{0}(\ell)=0, \quad x_{s_{2}}<x<\ell
$$

is

$$
v_{r}^{0}(x)=v_{2}^{0} \cosh ((x-\ell) / \lambda) .
$$

And finally, the solution of the first order right problem,

$$
\lambda^{2} v_{x x}^{1}=v^{1}-\tau v_{2}^{0} \cosh ((x-\ell) / \lambda), \quad v^{1}(\ell)=v_{2}^{1}, \quad v_{x}^{1}(\ell)=0, \quad x_{s_{2}}<x<\ell
$$

is

$$
v_{r}^{1}(x)=v_{2}^{1} \cosh ((x-\ell) / \lambda)-\frac{\tau v_{2}^{0}(x-\ell)}{2 \lambda} \sinh ((x-\ell) / \lambda)
$$

Matching the $v^{0}$ at $x_{s_{1}}$ and the $v^{1}$ at $x_{s_{1}}$, i.e.,

$$
v_{l}^{0}\left(x_{s_{1}}\right)=v_{m}^{0}\left(x_{s_{1}}\right) \quad \text { and } \quad v_{l}^{1}\left(x_{s_{1}}\right)=v_{m}^{1}\left(x_{s_{1}}\right)
$$

permits us to represent $a$ and $b$ in terms of $x_{s_{1}}$, and so the matching conditions at $x_{s_{2}}$, namely,

$$
v_{r}^{0}\left(x_{s_{2}}\right)=v_{m}^{0}\left(x_{s_{2}}\right) \quad \text { and } \quad v_{r}^{1}\left(x_{s_{2}}\right)=v_{m}^{1}\left(x_{s_{2}}\right)
$$

then become two equations for the two unknown synaptic locations. As these equations don't yield much to visual inspection we shall keep them under wraps. Though their numerical solution via Newton's method from a wide variety of starting guesses consistently landed in a small neighborhood of the true $x_{s_{1}}$ and $x_{s_{2}}$ we do not have a mathematical proof that this system possesses but one solution. Nonetheless, with our (approximate) $x_{s_{1}}$ and $x_{s_{2}}$ we may now solve sideways for $v_{l}(x, t)$ and $v_{r}(x, t)$ and so arrive at a standard Dirichlet problem for $v_{m}$, namely

$$
\begin{aligned}
\lambda^{2} v_{x x} & =\tau v_{t}+v, \quad x_{s_{1}}<x<x_{s_{2}} \\
v\left(x_{s_{1}}, t\right) & =v_{l}\left(x_{s_{1}}, t\right) \\
v\left(x_{s_{2}}, t\right) & =v_{r}\left(x_{s_{2}}, t\right) \\
v(x, 0) & =0
\end{aligned}
$$

Solving this for $v_{m}$ we then proceed to

$$
\begin{aligned}
& g_{1}(t)=\lambda^{2} \frac{\partial_{x} v_{m}\left(x_{s_{1}}, t\right)-\partial_{x} v_{l}\left(x_{s_{1}}, t\right)}{v_{l}\left(x_{s_{1}}, t\right)-E_{1}} \\
& g_{2}(t)=\lambda^{2} \frac{\partial_{x} v_{r}\left(x_{s_{2}}, t\right)-\partial_{x} v_{m}\left(x_{s_{2}}, t\right)}{v_{r}\left(x_{s_{2}}, t\right)-E_{2}}
\end{aligned}
$$


We illustrate this method, see fig. 6, on the cell above with synaptic inputs

$$
\begin{aligned}
& g_{1}(t)=(0.06) t^{4} \exp (-2 t) \quad \text { at } \quad x_{s_{1}}=0.035 \text { and } \\
& g_{2}(t)=(0.02) \max (0, t-2)^{3} \exp (-(t-2)) \text { at } x_{s_{2}}=0.0764
\end{aligned}
$$

with associated reversal potential $E_{1}=E_{2}=50$.

Regarding the recovery of more than 2 synapses let us call a set of voltage recordings separating if there is exactly one synapse between every pair of recording sites. With this, it is not difficult to prove

Proposition 2. The location and time courses of $N$ synapses onto a uniform sealed passive fiber are constructively determined by $N+1$ separating voltage recordings. The synaptic locations are recovered by solving $N$ nonlinear equations in the first $N$ moments of the $N$ potential measurements.

If the cell is nonuniform or not passive then we may not explicitly propagate these potential and current measurements. This can however be done numerically.

\section{Active Cells}

We now extend our findings to encompass cells with voltage dependent channels. We adopt the formalism of Hodgkin and Huxley and consider

$$
\begin{aligned}
\frac{d}{4 R_{i}} v_{x x} & =C_{m} v_{t}+I_{\mathrm{ion}}(v, w)+g_{s}(t) \delta\left(x-x_{s}\right)\left(v-E_{s}\right) . \\
I_{\mathrm{ion}}(v, w) & =g_{N a} w_{1}^{3} w_{2}\left(v-E_{N a}\right)+g_{K} w_{3}^{4}\left(v-E_{K}\right)+g_{C l}\left(v-E_{C l}\right) \\
\partial_{t} w_{j} & =\alpha_{j}(v)\left(1-w_{j}\right)-\beta_{j}(v) w_{j}, \quad j=1,2,3 \\
v(x, 0) & =v_{x}(0, t)=v_{x}(\ell, t)=0
\end{aligned}
$$

where $v$, and each of the reversal potentials, is with respect to rest. As above, the objective is to determine the location, $x_{s}$, and time course $g_{s}$, from knowledge of $v$ in time at $x=0$ and $x=\ell$. As above, we recognize that (5.1), together with the end potentials, can be written as an initial value problem in space on either side of $x_{s}$. The difficulty in this setting is the robust numerical implementation of the time derivatives. We again follow the the lead of Elden et al. by writing (5.1) as a first order system and then use a filtered discrete Fourier Transform to approximate $\partial_{t}$. In particular, for $x<x_{s}$ we study

$$
\begin{aligned}
v_{x} & =\left(4 R_{i} / d\right) u \\
u_{x} & =C_{m} v_{t}+I_{i o n}(v, w) \\
\partial_{t} w_{j} & =\alpha_{j}(v)\left(1-w_{j}\right)-\beta_{j}(v) w_{j}, \quad j=1,2,3
\end{aligned}
$$


by first advancing the gating the variables, $w_{j}$, in time via backward Euler and then advancing $u$ and $v$ in space via hybrid Euler. More precisely, starting from the discretization

$$
\begin{aligned}
\frac{v(x+d x, t)-v(x, t)}{d x} & =\left(4 R_{i} / d\right) u(x, t) \\
\frac{u(x+d x, t)-u(x, t)}{d x} & =C_{m} v_{t}(x, t)+I_{\text {ion }}(v(x, t), w(x, t)) \\
\frac{w_{j}(x, t+d t)-w_{j}(x, t)}{d t}= & \alpha_{j}(v(x, t+d t))\left(1-w_{j}(x, t+d t)\right) \\
& -\beta_{j}(v(x, t+d t)) w_{j}(x, t+d t)
\end{aligned}
$$

and knowledge of $t \mapsto u(x, t), t \mapsto v(x, t)$ and $w_{j}(x, 0)$ at a given $x$ (starting with $x=0$ ) we

(1) Advance the gating variables at $x$ through time via

$$
w_{j}(x, t+d t)=\frac{w_{j}(x, t)+d t \alpha_{j}(v(x, t+d t))}{1+d t\left\{\alpha_{j}(v(x, t+d t))+\beta_{j}(v(x, t+d t))\right\}}
$$

(2) Advance $u$ and $v$ in space via

$$
\begin{aligned}
& v(x+d x, t)=v(x, t)+\left(4 R_{i} d x / d\right) u(x, t) \\
& u(x+d x, t)=u(x, t)+d x\left\{C_{m} v_{t}(x, t)+I_{i o n}(v(x, t), w(x, t))\right\}
\end{aligned}
$$

where, in order to suppress the propagation of high frequency errors we approximate the time derivative of $v$ via

$$
v_{t}(x, t) \approx \frac{1}{2 \pi} \int_{-\omega_{c}}^{\omega_{c}}-i \omega \exp (i \omega t) \int_{-\infty}^{\infty} v(x, s) \exp (-i \omega s) d s d \omega
$$

where, as above, the Fourier Transform and its inverse are implemented in Matlab via the fast Fourier Transform.

This algorithm now permits us to march in space from known information gathered at the left boundary toward the (unknown) location of the synapse. A very similar algorithm, differing only in the initial discretization

$$
\begin{aligned}
& \frac{v(x, t)-v(x-d x, t)}{d x}=\left(4 R_{i} / d\right) u(x, t) \\
& \frac{u(x, t)-u(x-d x, t)}{d x}=C_{m} v_{t}(x, t)+I_{\text {ion }}(v(x, t), w(x, t))
\end{aligned}
$$

permits us to march leftward from known data. The synapse location will be that $x$ at which the two marching schemes produce the same $v$. More precisely, if $v_{L}$ and $v_{R}$ denote 
those solutions propagated from the left and right respectively from end potentials sampled at $K$ instants of time, then $x_{s}$ will be that point $x$ at which the mismatch

$$
\sum_{k=1}^{K}\left|v_{L}(x, k d t)-v_{R}(x, k d t)\right|^{2}
$$

is least. With $x_{s}$ in hand one finds, as above, that integrating the $v$ equation in (5.1) in space about $x_{s}$ produces

$$
g_{s}(t)=\frac{d}{4 R_{i}} \frac{v_{x}\left(x_{s}^{+}, t\right)-v_{x}\left(x_{s}^{-}, t\right)}{v\left(x_{s}, t\right)-E_{s}} .
$$

Moreover, this synaptic potential, $v\left(x_{s}, t\right)$, and the two currents, $v_{x}\left(x_{s}^{+}, t\right)$ and $v_{x}\left(x_{s}^{-}, t\right)$ are immediate byproducts of our space marching schemes. We illustrate this method, see fig. 7 , on a cell with geometry and passive parameters as described by (2.5), and standard squid axon channels and kinetics

$$
\begin{aligned}
\alpha_{m}(v) & =\frac{25-v}{10(\exp ((25-v) / 10)-1)} \quad \text { and } \quad \beta_{m}(v)=4 \exp (-v / 18), \\
\alpha_{h}(v) & =0.07 \exp (-v / 20) \quad \text { and } \quad \beta_{h}(v)=\frac{1}{\exp ((30-v) / 10)+1}, \\
\alpha_{n}(v) & =\frac{10-v}{100(\exp (1-v / 10)-1)} \quad \text { and } \quad \beta_{n}(v)=0.125 \exp (-v / 80), \\
E_{K} & =-6, E_{N a}=127, E_{C l}=2.85 \quad \mathrm{mV} \\
G_{K} & =36, G_{N a}=120, G_{C l}=0.3 \quad \mathrm{mS} / \mathrm{cm}^{2}
\end{aligned}
$$

with a synapse at $x_{s}=0.039 \mathrm{~cm}$ with conductance

$$
g_{s}(t)=(7.5 \mathrm{e}-4) \max (0, t-2)^{2} \exp (1-t / 2) \quad m S
$$

and reversal potential $E_{s}=50 \mathrm{mV}$. We corrupted the end potentials with multiplicative Gaussian noise, see fig. $7(\mathrm{~B})$, and then applied a $4 k H z$ low pass filter prior to their sideways propagation. We experimented with a number of cutoff values in the filtered differentiator, (5.2), and found $\omega_{c} \approx 1 k H z$ to give the best performance.

With regard to fig. $7(\mathrm{C})$, if the mismatch does not achieve a small, well defined, minimum then it is likely that the fiber has recently synaptic input at more than one site. In this case, supposing their were two sites, one ought to take two additional potential recordings at locations that separate the actual synaptic sites. One may then solve (5.1) between these two locations and so recover the two currents there. This would then permit 
one to propagate the new left data to the left and new right data to the right until the associated mismatches are made small. Of course if one of these mismatches does not get small then additional separating recordings must be made.

It is clear from fig. 7 that (5.6) is a subthreshold stimulus and that, given the uniform excitability of the cell, an increase in amplitude of $g_{s}$ will elicit a bidirectional action potential at $x_{s}$. In such a case we may recover the synapse location $x_{s}$ and the initial time, $t_{s}$, of the conductance change but we can not decipher from the end potentials anything more about the time course of the synaptic change. More precisely, if $c$ denotes the known wave speed and $t_{0}$ and $t_{\ell}$ denote the times at which the respective end potentials begin to deviate from rest then $c\left(t_{0}-t_{s}\right)=x_{s}$ and $c\left(t_{\ell}-t_{s}\right)=\ell-x_{s}$ and so

$$
t_{s}=\frac{t_{0}+t_{\ell}}{2}-\frac{\ell}{2 c} \quad \text { and } \quad x_{s}=\frac{\ell-c\left(t_{\ell}-t_{0}\right)}{2}
$$

mark the time and place of synaptic input. We offer a concrete example in fig. 8. Scaling the $g_{s}$ of $(5.6)$ by $3 / 2$ elicits the bidirectional action potential of fig. 8(A). Its speed $c \approx$ $0.03 \mathrm{~cm} / \mathrm{ms}$. Regarding associated end potentials, fig. 8(B), although their "start times" are a bit difficult to discern there is clearly a phase difference $t_{\ell}-t_{0} \approx 1 \mathrm{~ms}$ and so the estimated synaptic location is $x_{s} \approx 0.035 \mathrm{~cm}$. These end potentials are stereotypical action potentials that show little stimulus artifact and indeed their rapid rise and fall defeats the recovery algorithm outlined above.

\section{Discussion}

We have demonstrated that in theory and in simulated practice one may faithfully read the location and time course of synaptic conductance from multi-site potential recordings in a branched, fully active cell. The intrusion of known spatial nonuniformity, whether via spines, tapering and/or heterogeneous channel distribution complicates but in no way precludes the success of the space marching schemes of the previous section. We have not conducted a systematic determination of the sensitivity of our methods to errors in potential recordings and/or uncertainties in the cell's passive and active properties but instead have offered up typical illustrations of their performance on simulated data. It remains, of course, to test these tools on true experimental data.

Regarding existing methods, our method is not limited to the AMPA-NMDA pairing of (Kleppe \& Robinson, 1999) and, unlike (Häusser \& Roth, 1997), does not require multiple trials. Each of the three methods rely on accurate voltage and/or current traces as 
well as knowledge of the cell morphology and passive parameters. Our method, however, is constructive and so this dependence is explicit while the two existing methods rely on black-box fitting routines. For example, the estimation of the synaptic site, $x_{s}$, via (3.10) depends in an obvious fashion on the length, $\ell$, and space constant, $\lambda$, and the ratio of the means, $v_{0}^{0} / v_{1}^{0}$, of the two end potentials. Similarly, we offer an explicit formula for the synaptic conductance, (3.11), in terms of $\lambda$, the synaptic potential and the two associated axial currents, each of which, in (3.14)-(3.15), we present explicitly in terms of the cell's passive parameters, $\mu(\omega)$, and the Fourier Transform of the two end potentials. Also, unlike (Häusser \& Roth, 1997) and (Kleppe \& Robinson, 1999), we show how to accommodate multiple synapses and excitable cells. This accommodation however places a significant burden on the experimentalist. For example, in the case of $N$ synapses, the proper placement of separating electrodes by hand is probably not feasible for $N>3$, and though the imaging apparatus of (Bullen \& Saggau, 1999) may be steered by our algorithm to the proper locations it is not obvious that the images yet offer the resolution necessary to quantify EPSPs. And finally, in order to account for active properties the salient currents must first be identified and then their respective kinetics and spatial distribution must be mapped before applying the algorithm of $\S 5$.

\section{References}

Bekkers JM, Stevens CF (1996) Cable properties of cultured hippocampal neurons determined from sucrose-evoked miniature EPSCs. J. Neurophysiol. 75(3): 1250-5.

Bernard C, Johnston D (2003) Distance-dependent modifiable threshold for action potential back-propagation in hippocampal dendrites. J. Neurophysiol. 90(3): 1807-16.

Bullen A, Saggau P (1999) High-speed, random-access fluorescence microscopy: II. Fast quantitative measurements with voltage-sensitive dyes. Biophys. J. 76(4) :2272-87.

Cox SJ, Griffith B (2001) Recovering Quasi-Active properties of dendrites from dual potential recordings, J. Computational Neuroscience, 11(2): 95-110.

Eldén, L, Berntsson, F, Regínska, T (2000) Wavelet and Fourier methods for solving the sideways heat equation. SIAM J. Sci. Comput. 21(6): 2187-2205

Hartline DK, Castelfranco AM (2003) Simulations of voltage clamping poorly spaceclamped voltage-dependent conductances in a uniform cylindrical neurite. J. Comput. Neurosci. 14: 253-269.

Häusser M, Roth A. (1997) Estimating the time course of the excitatory synaptic 
conductance in neocortical pyramidal cells using a novel voltage jump method. J. Neurosci. 17(20): 7606-25.

Inoue M, Hashimoto Y, Kudo Y, Miyakawa H (2001) Dendritic attenuation of synaptic potentials in the CA1 region of rat hippocampal slices detected with an optical method. Eur. J. Neurosci. 13(9): 1711-21.

Jack JJ, Miller S, Porter R, Redman SJ (1971) The time course of minimal excitory post-synaptic potentials evoked in spinal motoneurones by group Ia afferent fibres. J. Physiol. (Lond) 215: 353-380.

Jaffe DB, Carnevale NT (1999) Passive normalization of synaptic integration influenced by dendritic architecture. J. Neurophysiol. 82(6): 3268-85.

Johnston D, Brown TH (1983) Interpretation of voltage-clamp measurements in hippocampal neurons. J. Neurophysiol. 50: 464-486.

Kleppe IC, Robinson HP (1999) Determining the activation time course of synaptic AMPA receptors from openings of colocalized NMDA receptors. Biophys. J. 77(3): 141827.

Koch C, Douglas R, Wehmeier U (1990) Visibility of synaptically induced conductance changes: theory and simulations of anatomically characterized cortical pyramidal cells. J. Neurosci. 10(6): 1728-44.

Major G (1993) Solutions for transients in arbitrarily branching cables: III. Voltage clamp problems. Biophys. J. 65: 469-491.

Mainen ZF, Carnevale NT, Zador AM, Claiborne BJ, Brown TH (1996) Electrotonic architecture of hippocampal CA1 pyramidal neurons based on three-dimensional recon structions. J. Neurophysiol. 76: 1904-1923.

Pearce RA (1993) Physiological evidence for two distinct GABAA responses in rat hippocampus. Neuron 10: 189-200.

Rall W (1967) Distinguishing theoretical synaptic potentials computed for different soma-dendritic distributions of synaptic input. J. Neurophysiol. 30(5): 1138-1168

Rall W, Segev I (1985) Space-clamp problems when voltage clamping branched neurons with intracellular microelectrodes. In: Voltage and patch clamping with microelectrodes (Smith Jr TG, Lecar H, Redman SJ, Gage P, eds), 191-215. Bethesda, MD: American Physiological Society.

Spruston N, Jaffe DB, Williams SH, Johnston D (1993) Voltage- and space-clamp errors associated with the measurement of electrotonically remote synaptic events. J. 
Neurophysiol. 70: 781-802.

Stuart G, Spruston N (1998) Determinants of voltage attenuation in neocortical pyramidal neuron dendrites. J. Neurosci. 18(10): 3501-10.

Tuckwell, HC (1988) Introduction to theoretical neurobiology. Cambridge University Press, New York.

\section{Figure Legends}

Figure 1. Response of the model cell, (2.5), to the stimulus (2.6). (A) The full space-time response. Computed via finite differences in space, $d x=4 \mu m$, and backward Euler in time, $d t=10 \mu s$. (B) The associated soma potential, $v_{0}$.

Figure 2. Recovering the synaptic site, $y_{s}$. (A) The graph of $F$ (solid) and the level $c$ (dashed) stemming from (2.16) and associated with the cell described in (2.5) and the true synapse described in (2.6). (B) Same as panel (A) except that $\alpha=1.5$. (C) The solid trace is the soma response to the true synapse while the dashed trace is the soma response to the false synapse.

Figure 3. Computed response of the cell (2.5) to the synapse (3.4) using finite differences in space $(d x=4 \mu m)$ and backward Euler in time $(d t=10 \mu s)$. (A) The full space-time response. (B) The end potentials corrupted with multiplicative Gaussian noise.

Figure 4. (A) Determination of $x_{s}$ from (3.10) and the noisy end potentials of fig. 3(B). (B) The magnitude of the Fourier Transform of the $v_{0}$ from fig. 3(B). The bulk of the energy lies at frequencies between $\pm 4 k H z$. (C) Determination of $g$ via (3.11) using a cutoff frequency of $\omega_{c}=4 \mathrm{kHz}$. The recovered $g$ is so close to the true that we have plotted the true along with the difference between the true and recovered.

Figure 5. A simple branched cell.

Figure 6. Recovering two locations and two synaptic time courses from potentials measured at 3 locations in the cell described by (2.5) and subject to (4.10). (A) The full space-time response. (B) The three corrupted voltage measurements taken at $x=0, x=0.047$ and $x=0.1$. (C) The true, fast and early, $g_{1}$ and the error accrued in its recovery via (4.9). (D) The true, slow and late, $g_{2}$ and the error accrued in its recovery via (4.9).

Figure 7. Recovery of $x_{s}$ and $g_{s}$ when the cell described by (2.5) and (5.5) is subjected to (5.6). (A) The full space-time response. The space-like curves (those parallel to the 
$x$-axis) were obtained from the standard hybrid-Euler (backward on the linear terms on forward on the nonlinear terms) solution to (5.1) with $d t=50 \mu \mathrm{s}$ and $d x=4 \mu \mathrm{m}$. The time-like curves (those parallel to the $t$-axis) were obtained by the sideways solution to (5.1) commencing with filtered corrupted versions of the end potentials arising from the forward solution. We have not plotted the propagated solutions past the synapse (as they begin to lose coherence). (B) The corrupted end potentials. (C) Determination of $x_{s}$ as the minimizer of the mismatch in (5.3). (D) The true (solid) $g_{s}$ and that recovered (dashed) via (5.4) upon propagating filtered versions of the corrupted potentials in pane (B).

Figure 8. Response to suprathreshold synaptic input. (A) The solution of (5.1) with $g_{s}$ multiplied by $3 / 2$. (B) The associated end potentials. 
Figure 1(A)

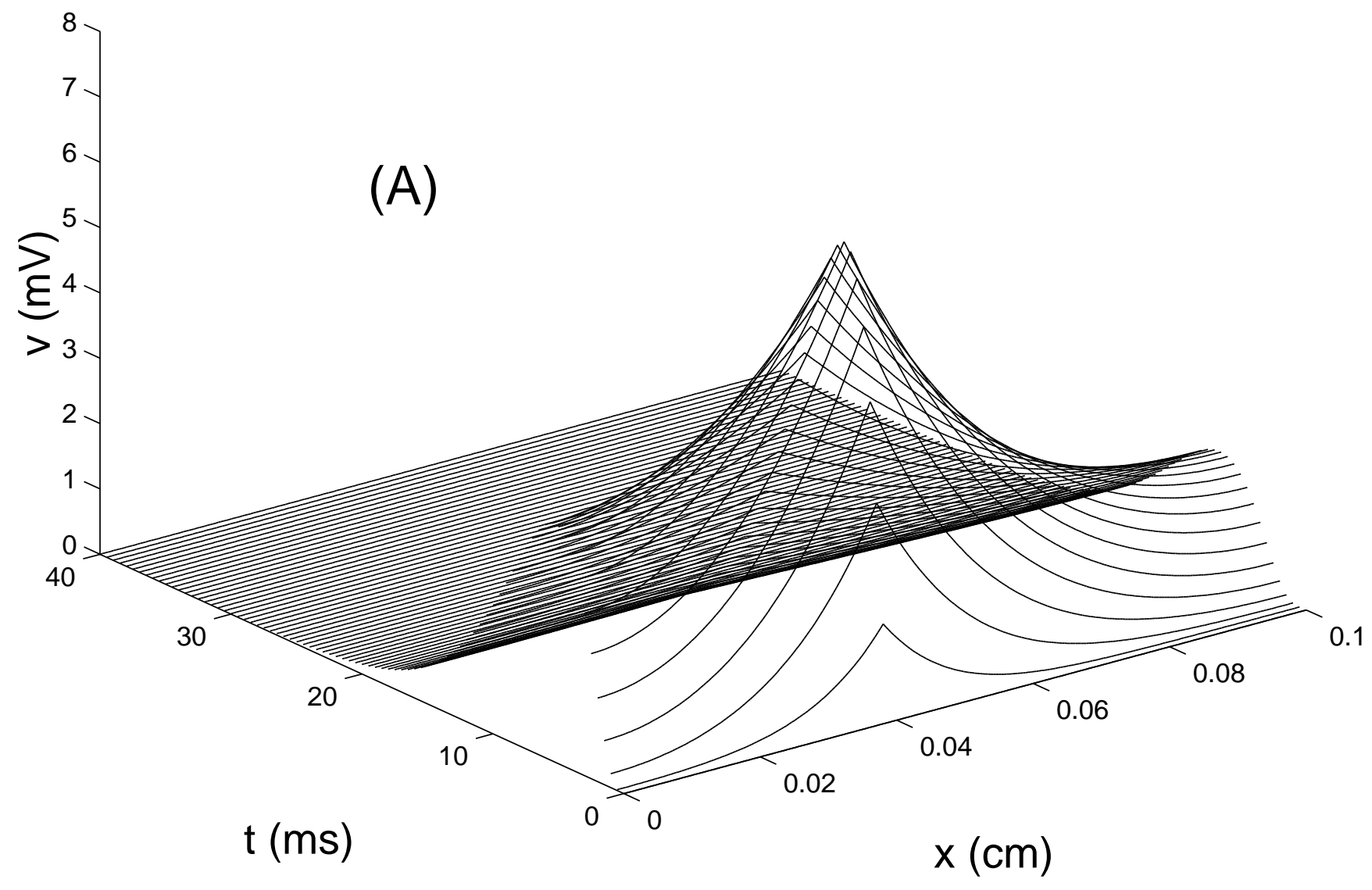


Figure 1(B)

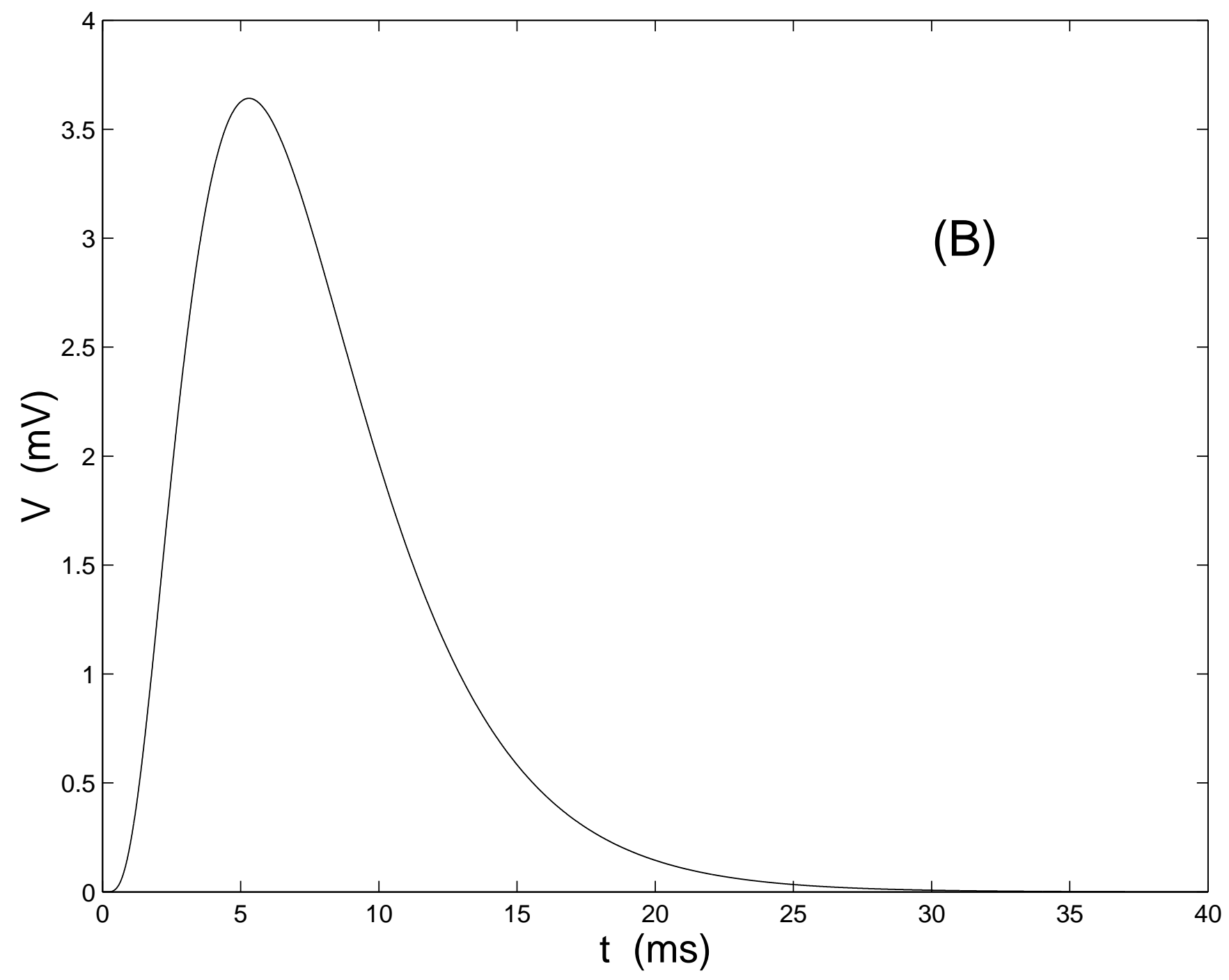


Figure 2(A)

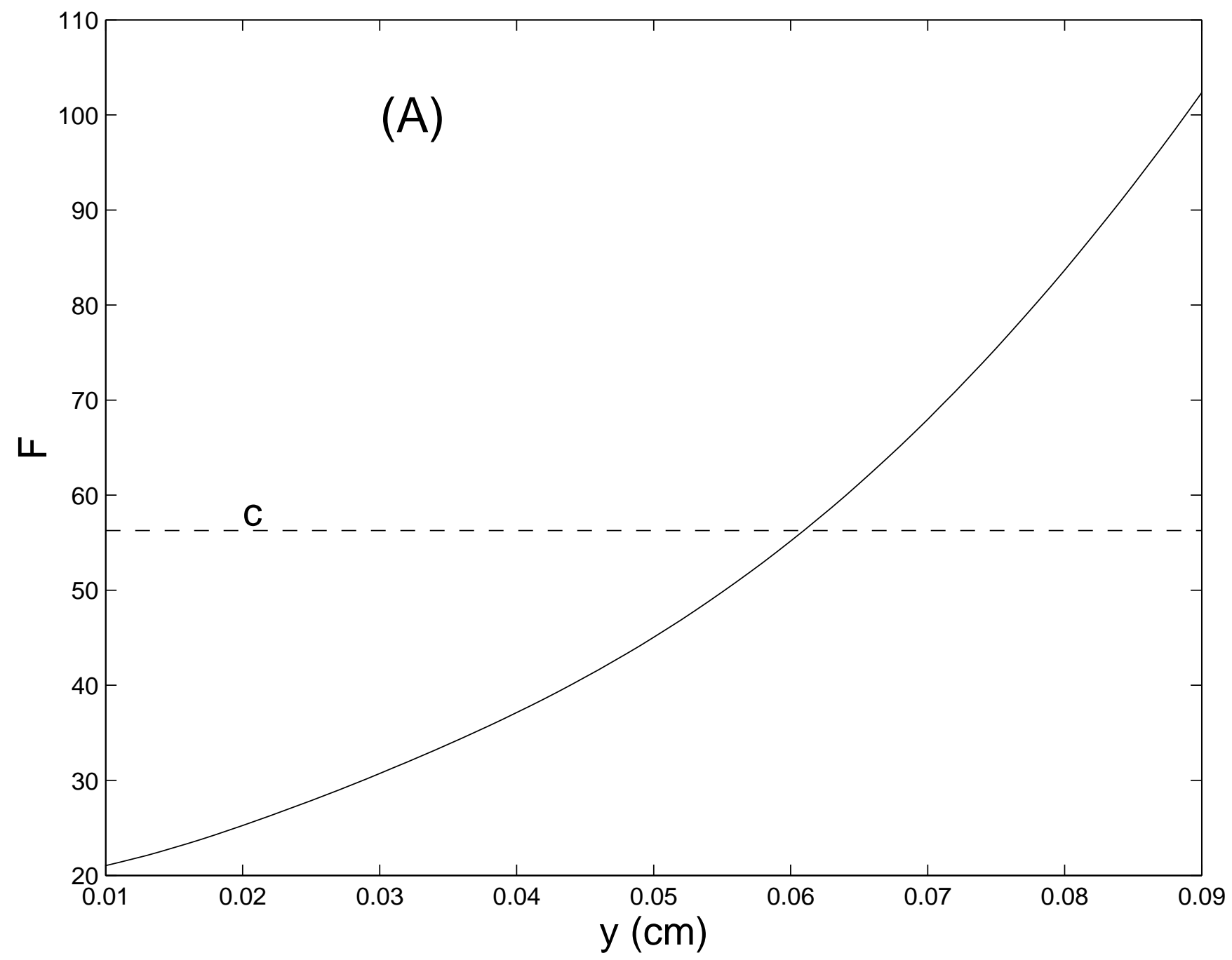


Figure 2(B)

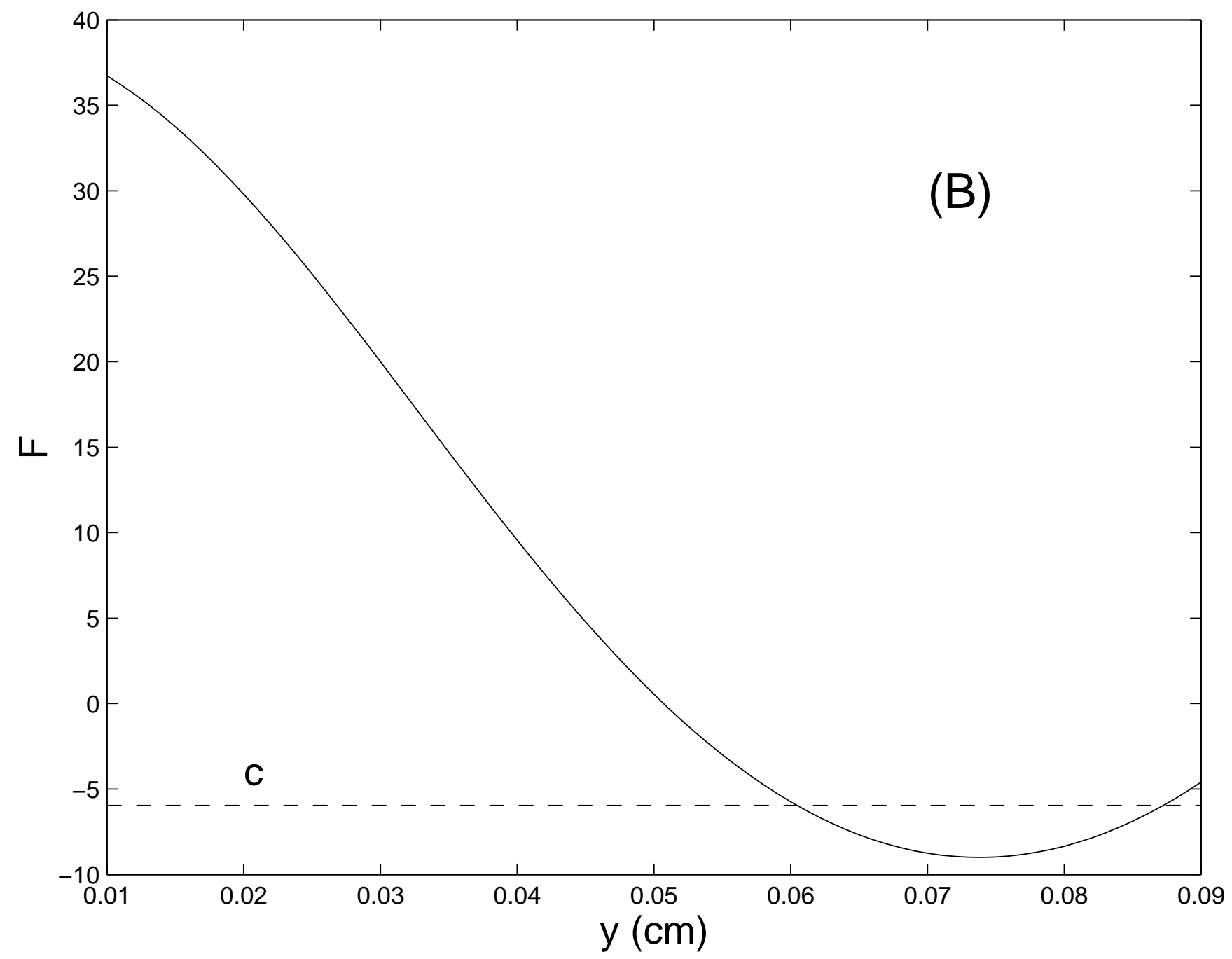


Figure 2(C)

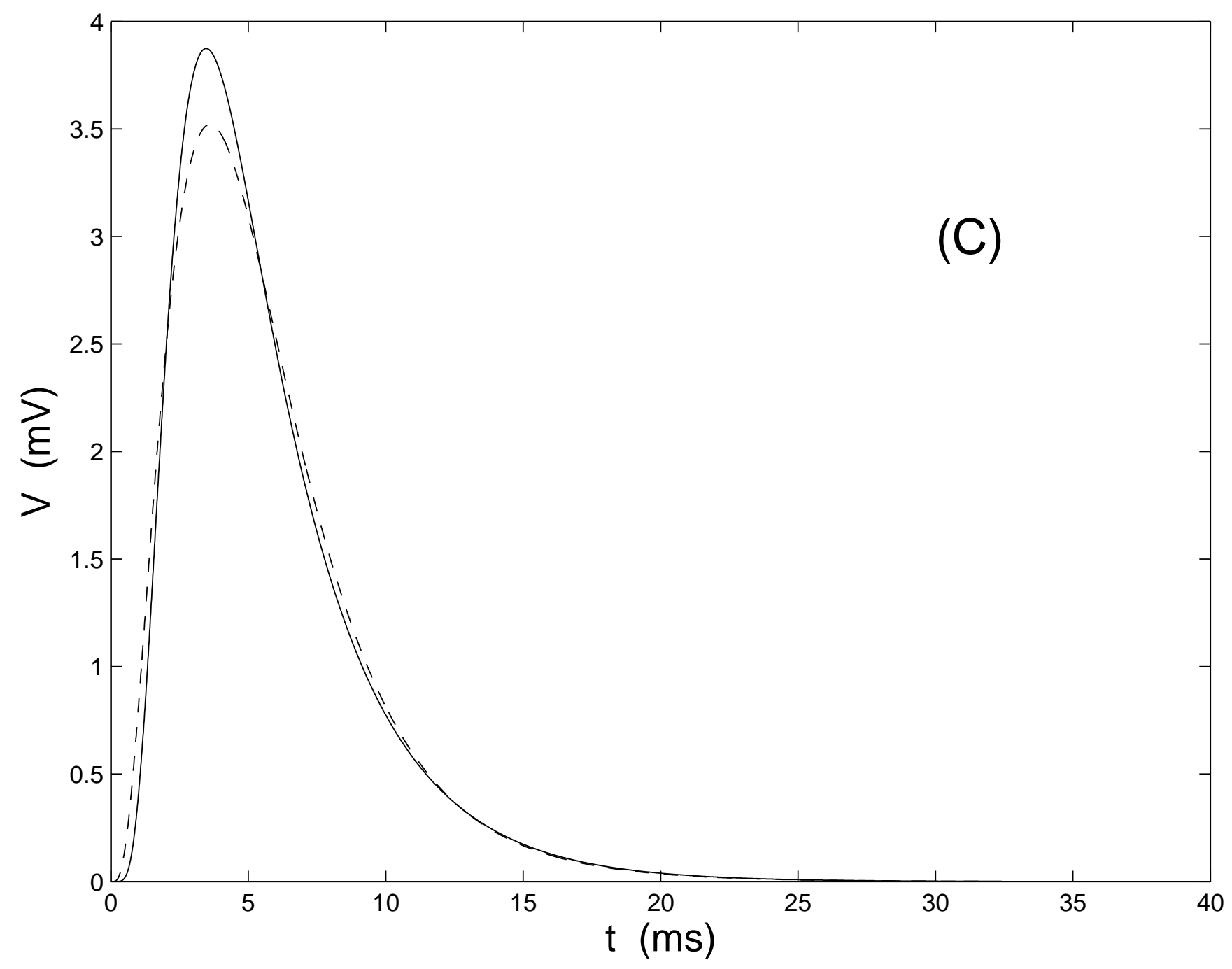


Figure $3(\mathrm{~A})$

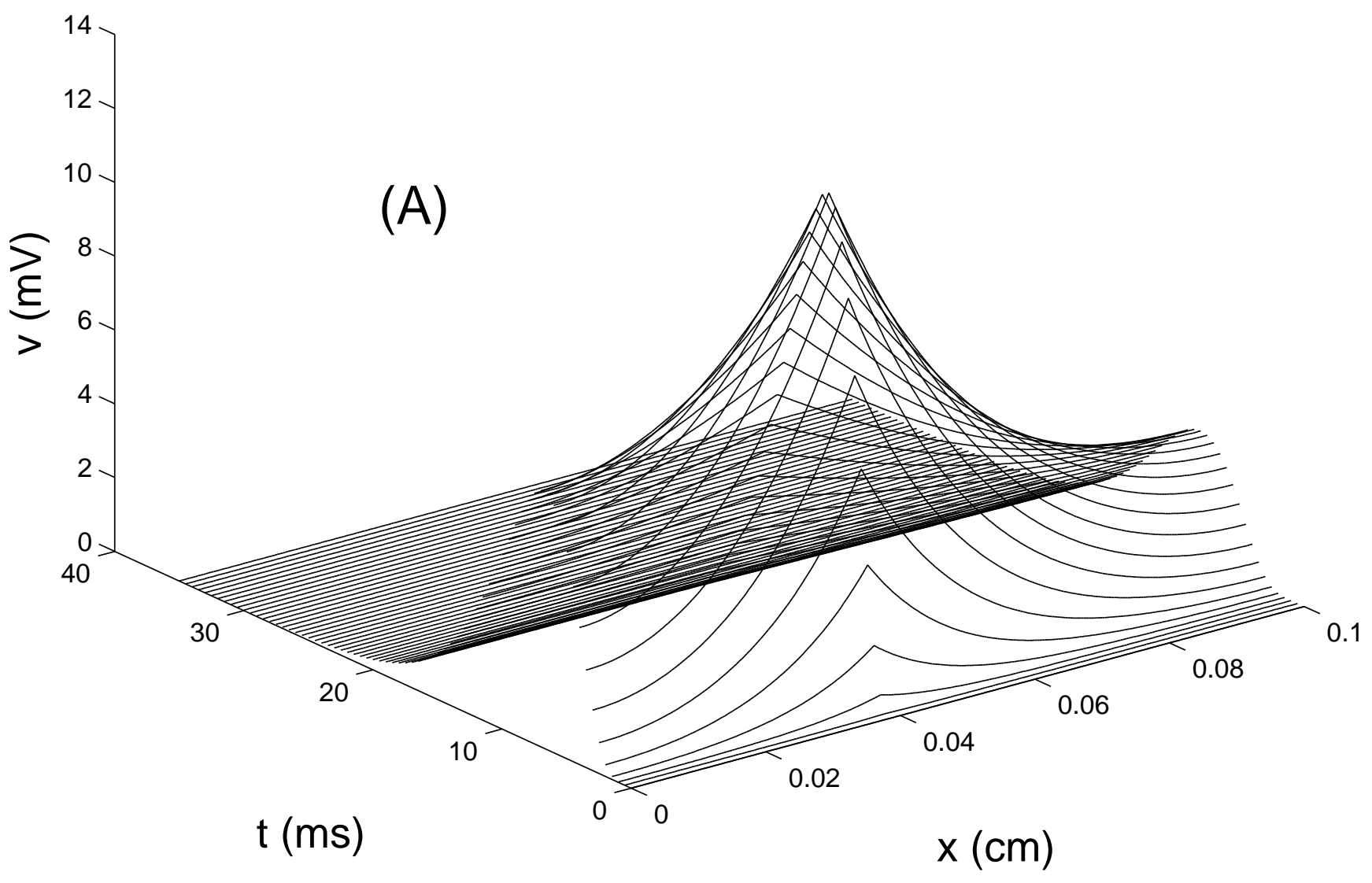


Figure 3(B)

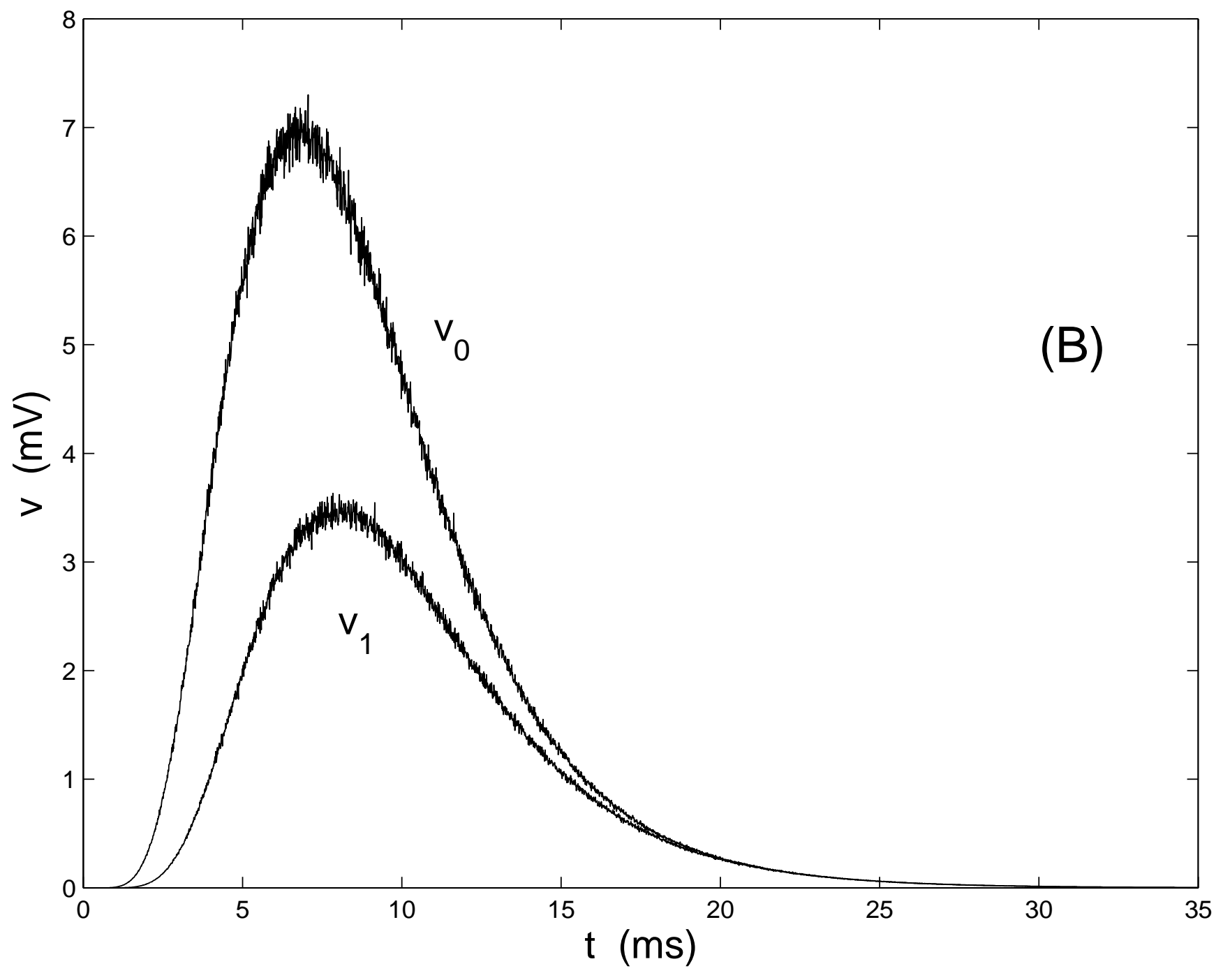


Figure 4(A)

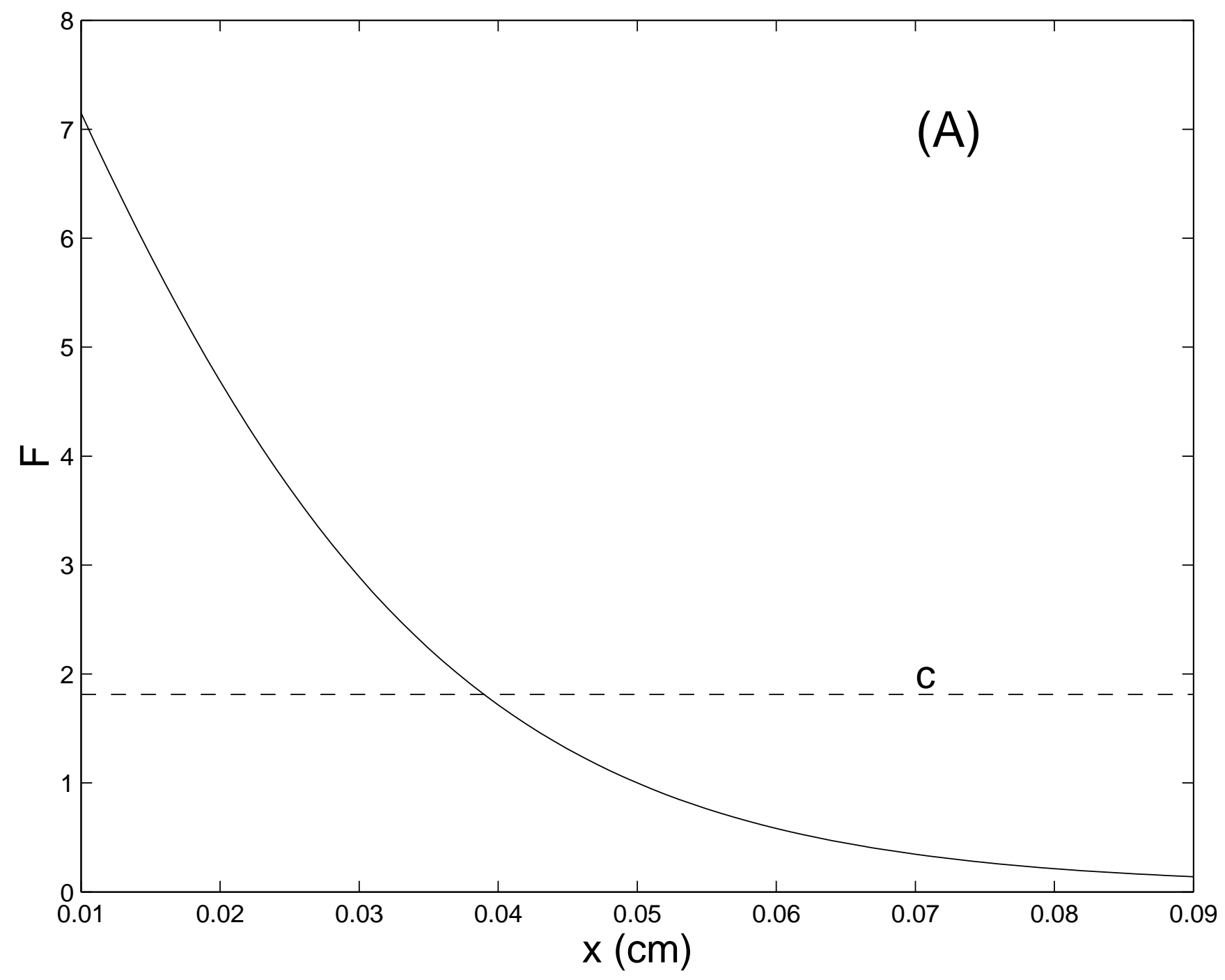


Figure 4(B)

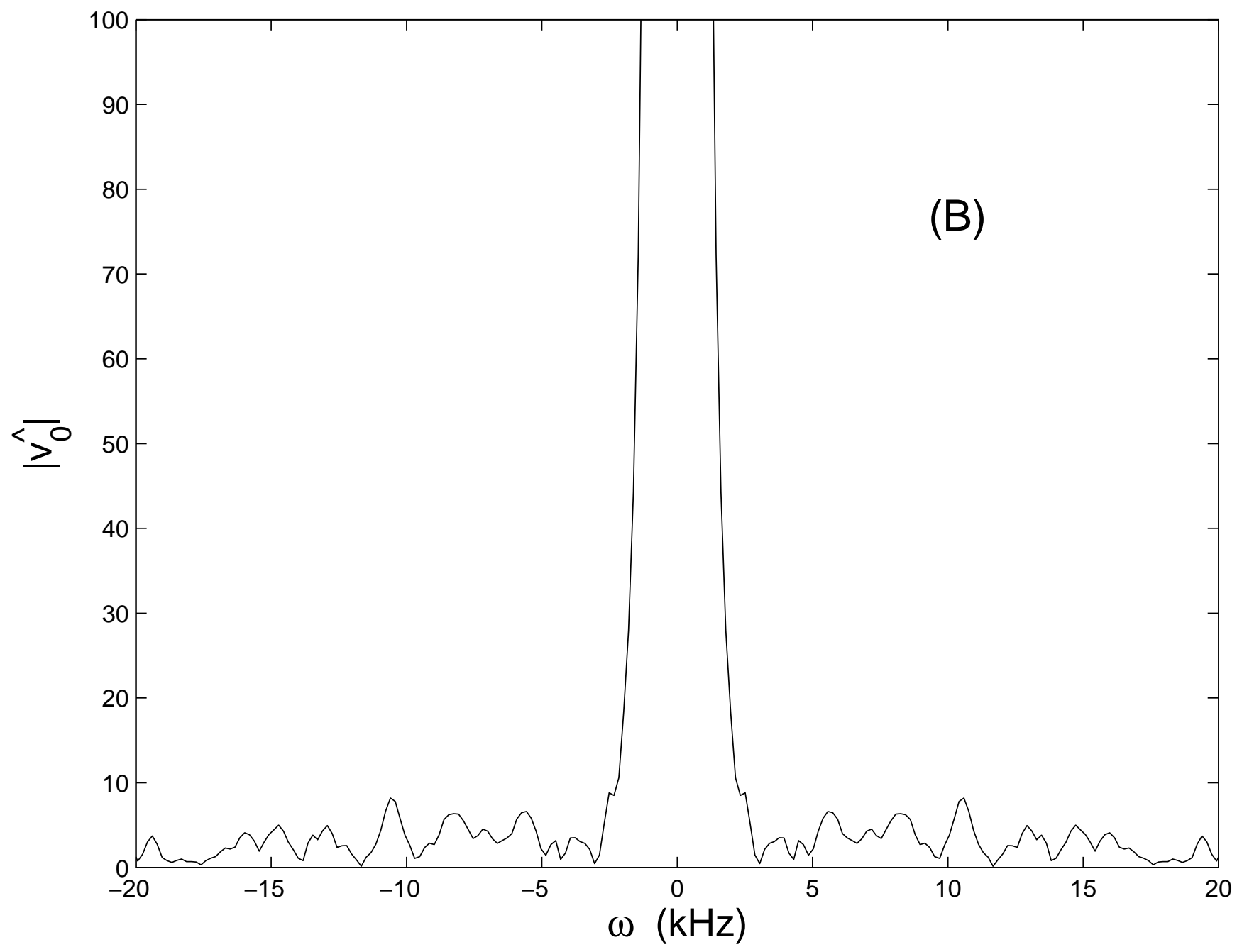


Figure 4(C)

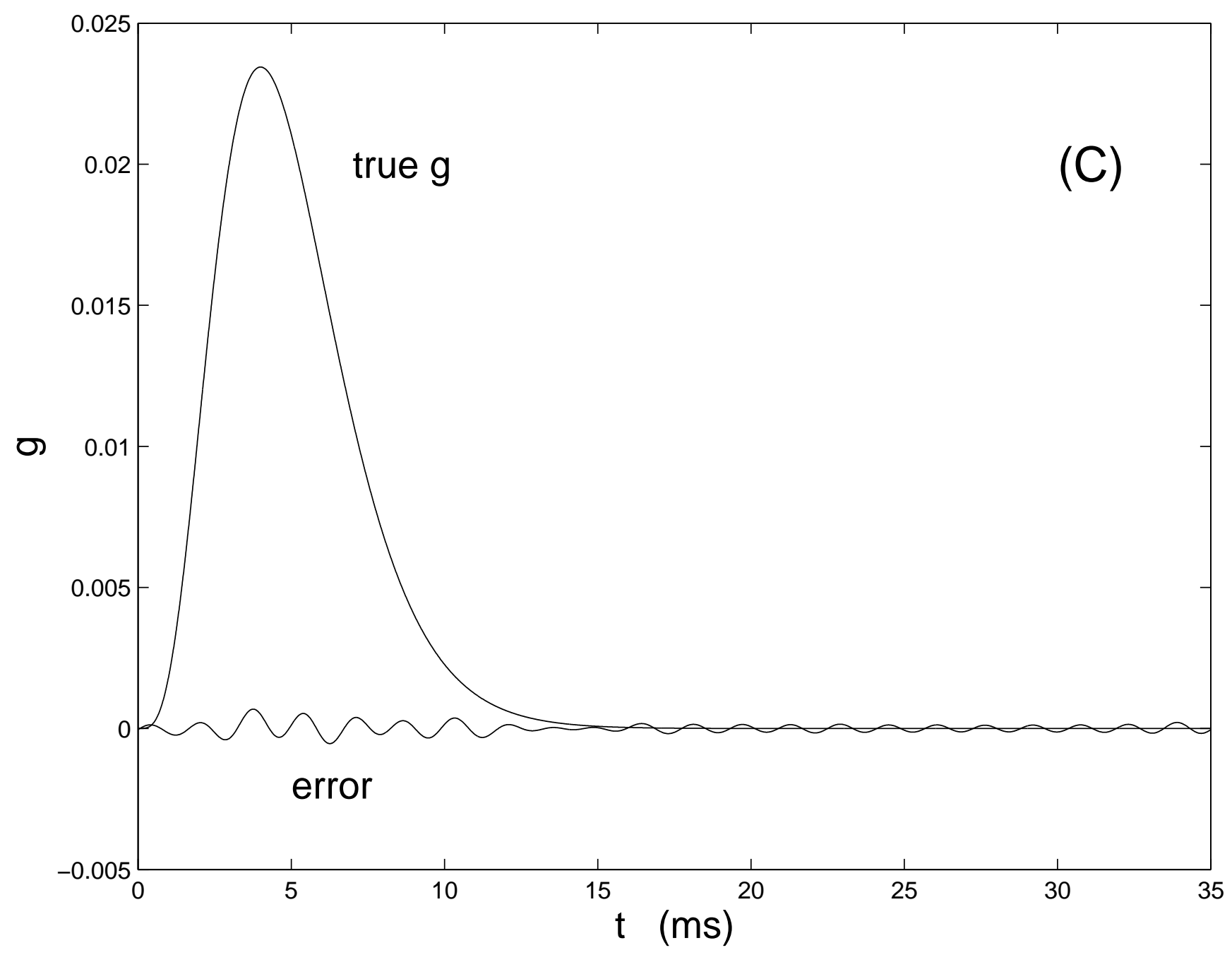


Figure 5

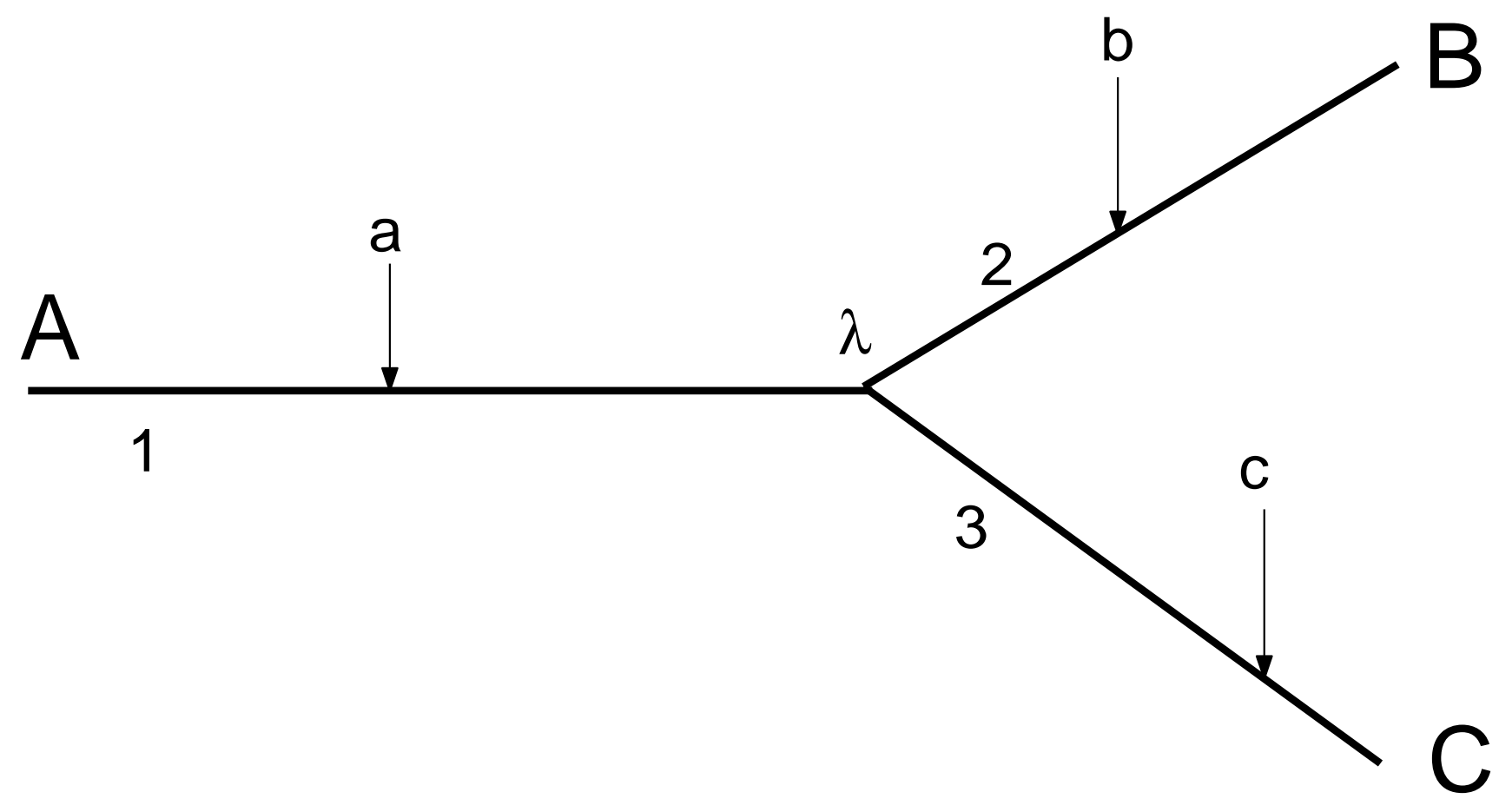


Figure 6(A)

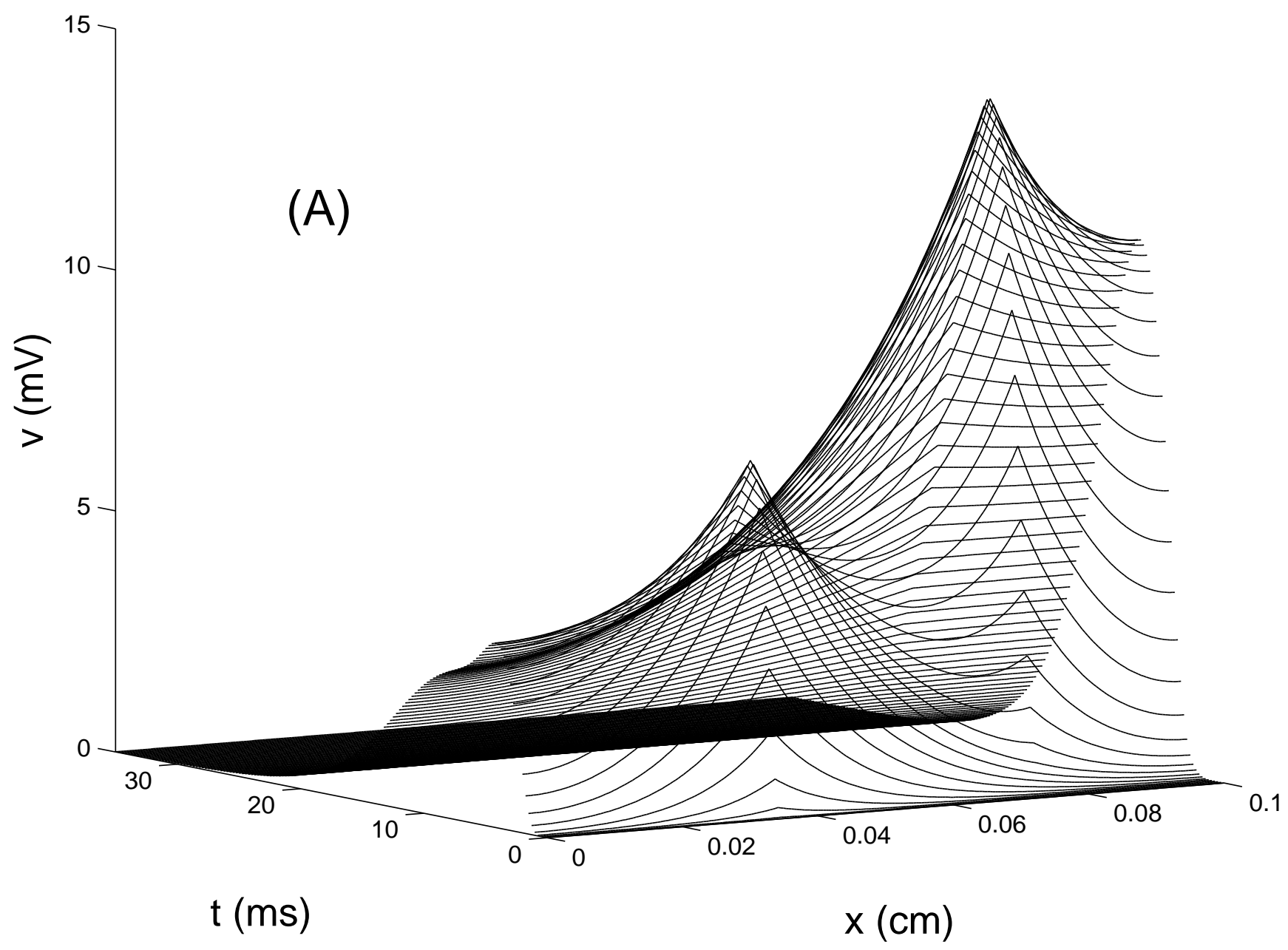


Figure 6(B)

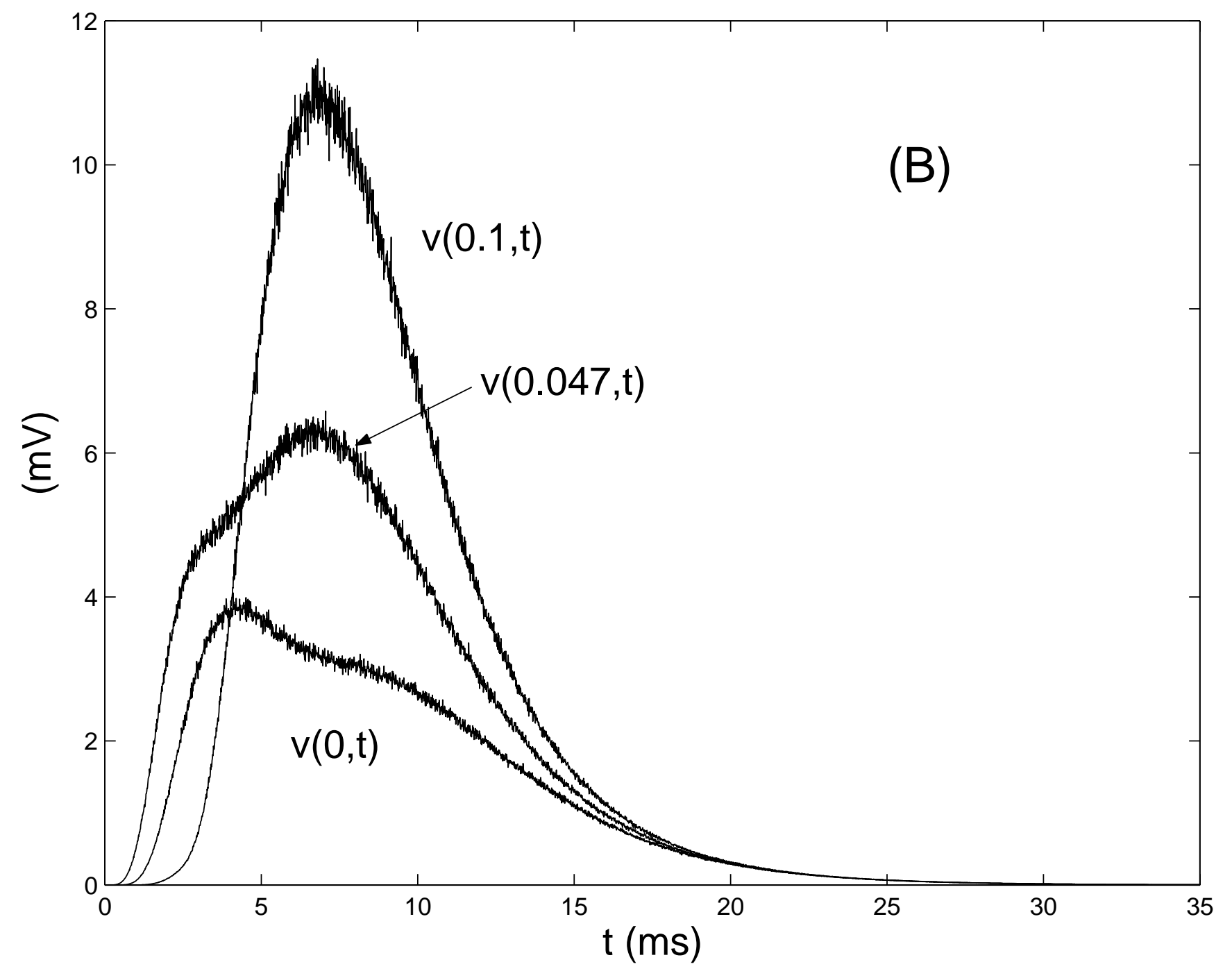


Figure 6(C)

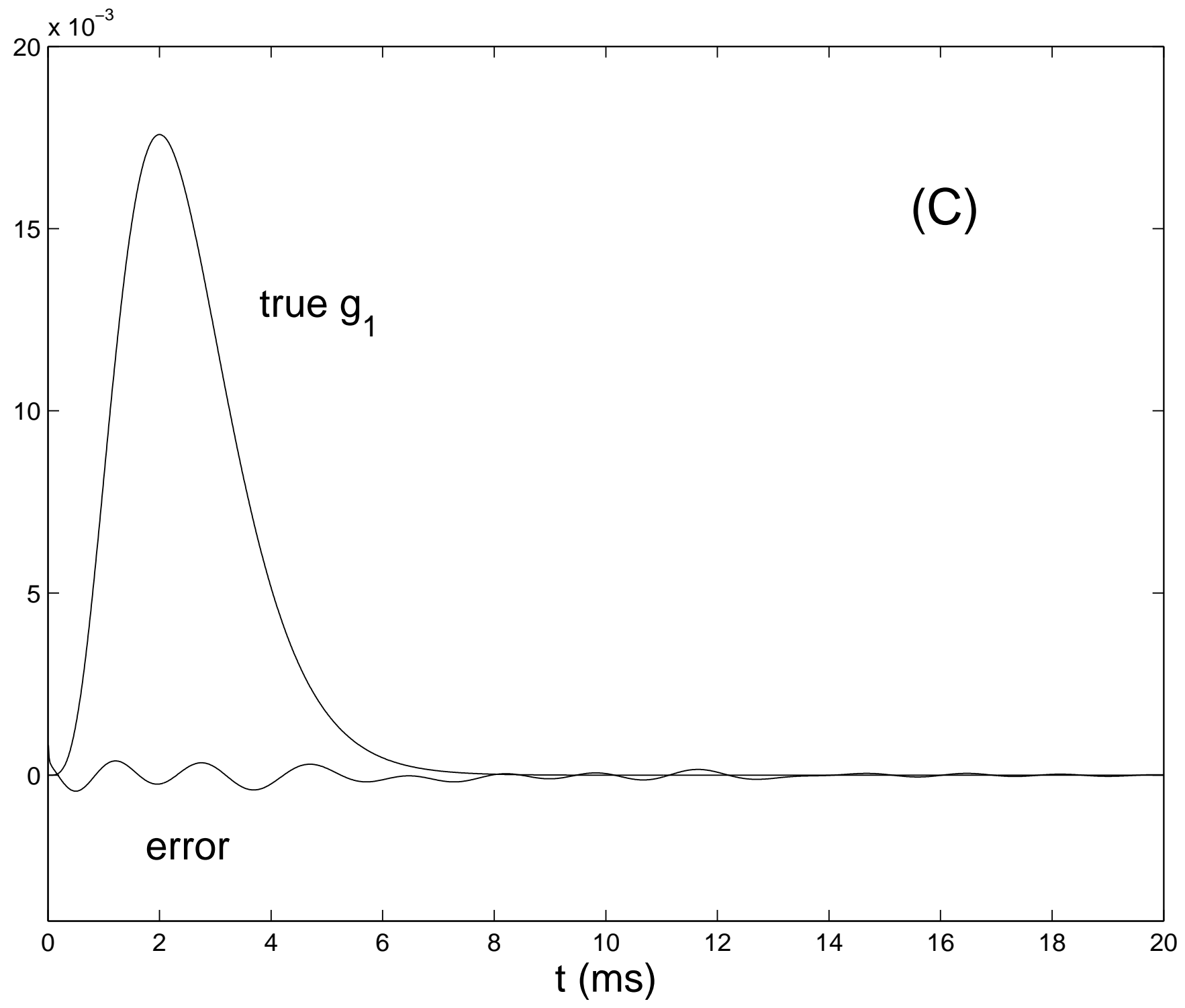


Figure 6(D)

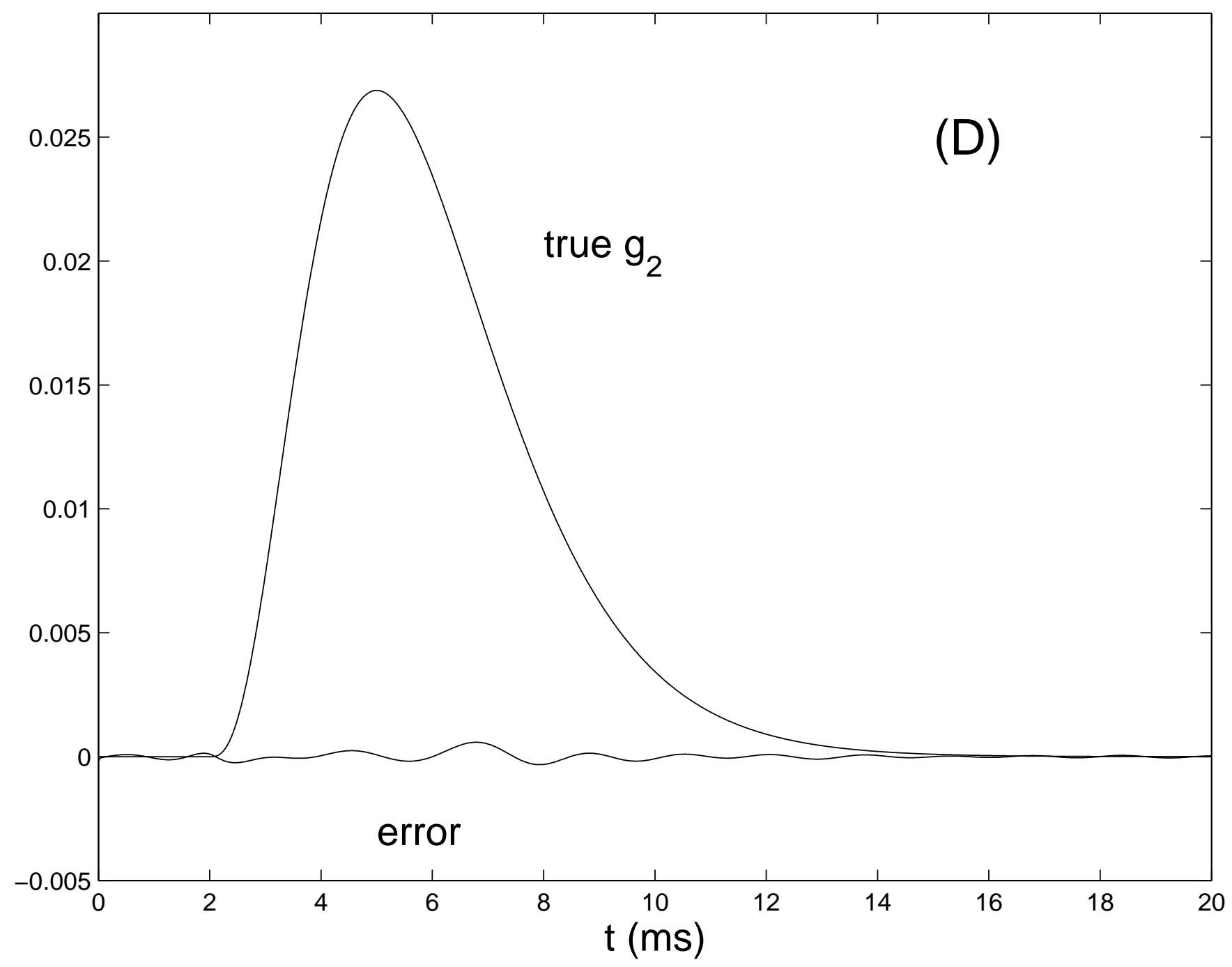


Figure $7(\mathrm{~A})$

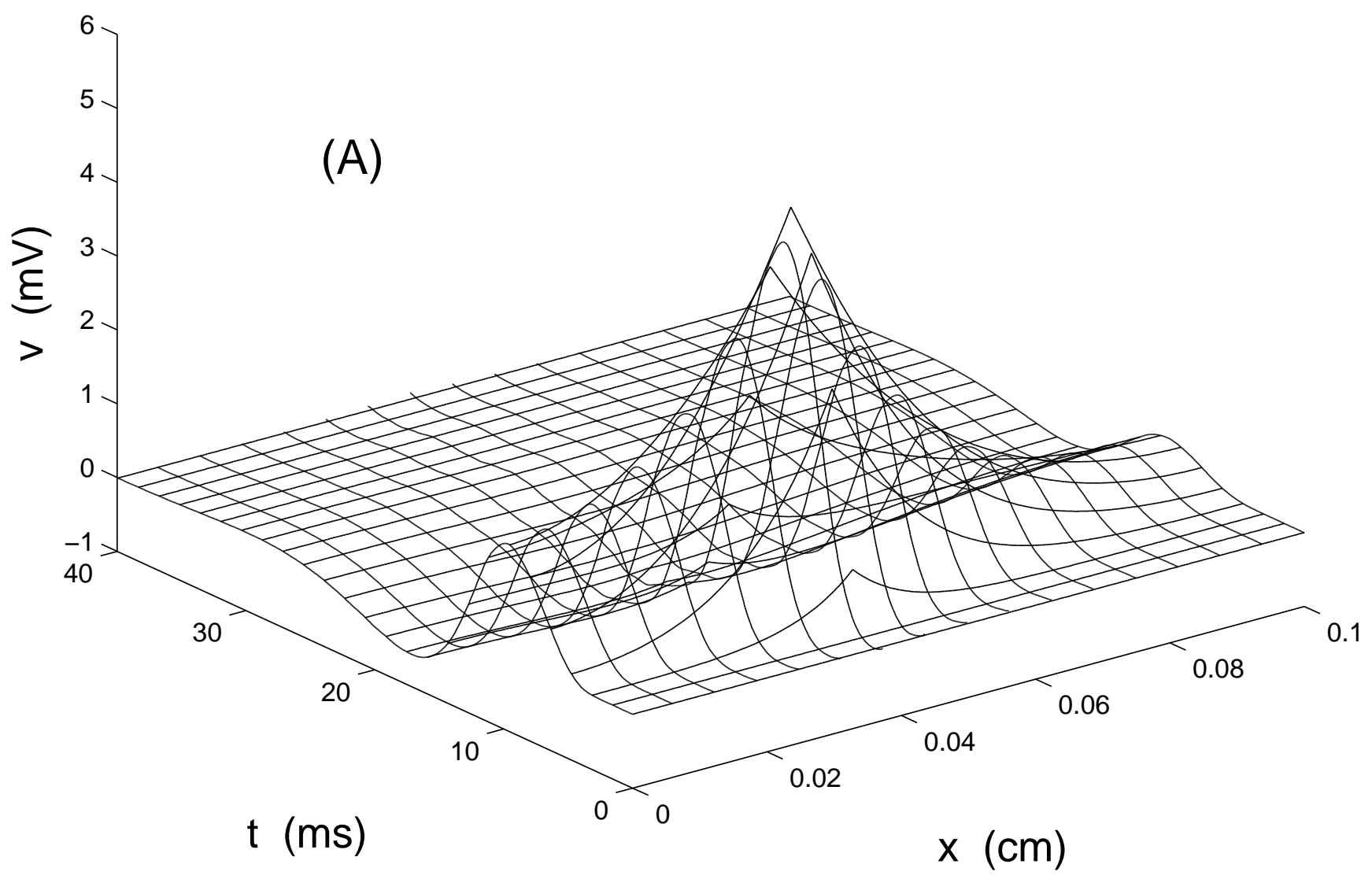


Figure 7(B)

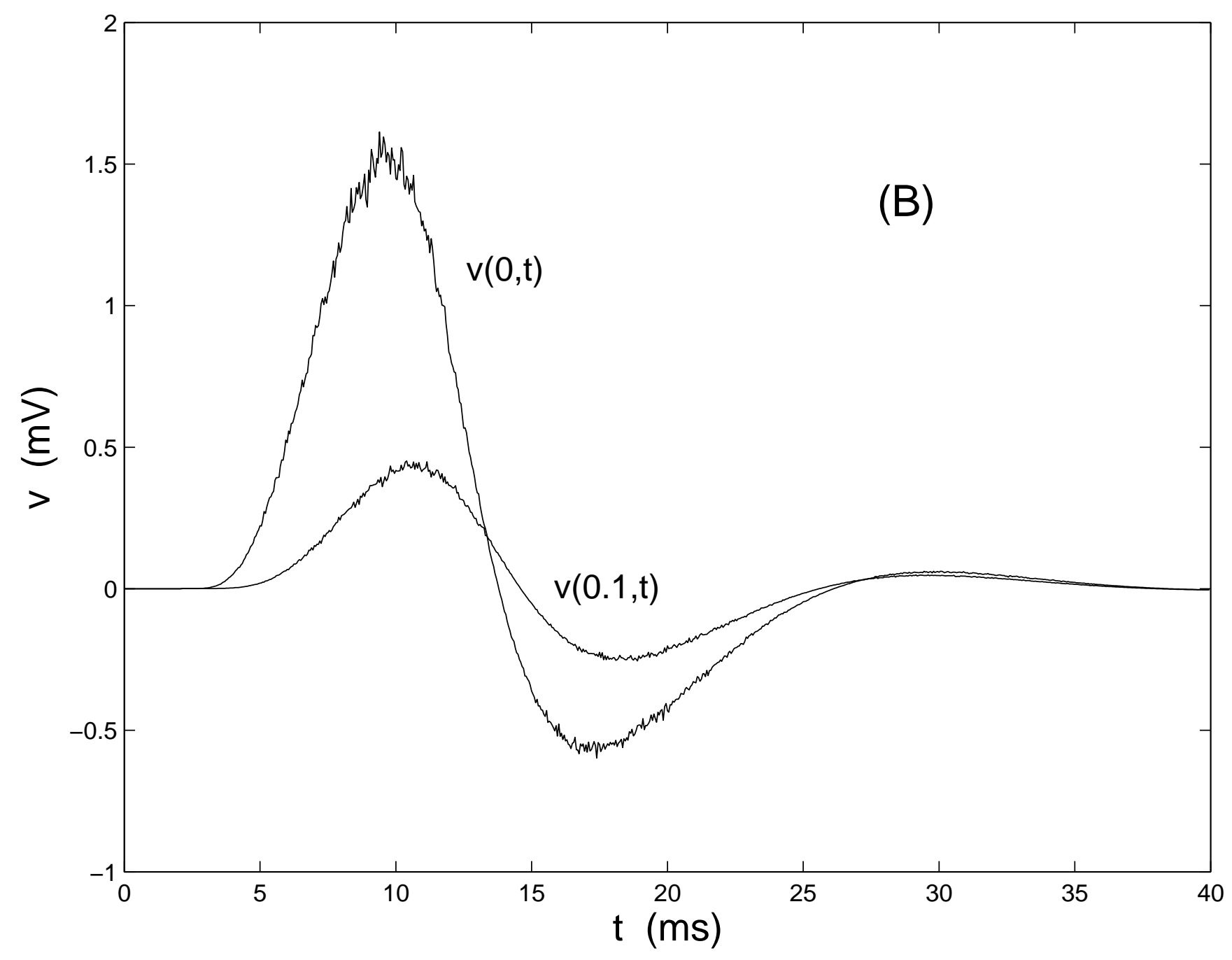


Figure 7(C)

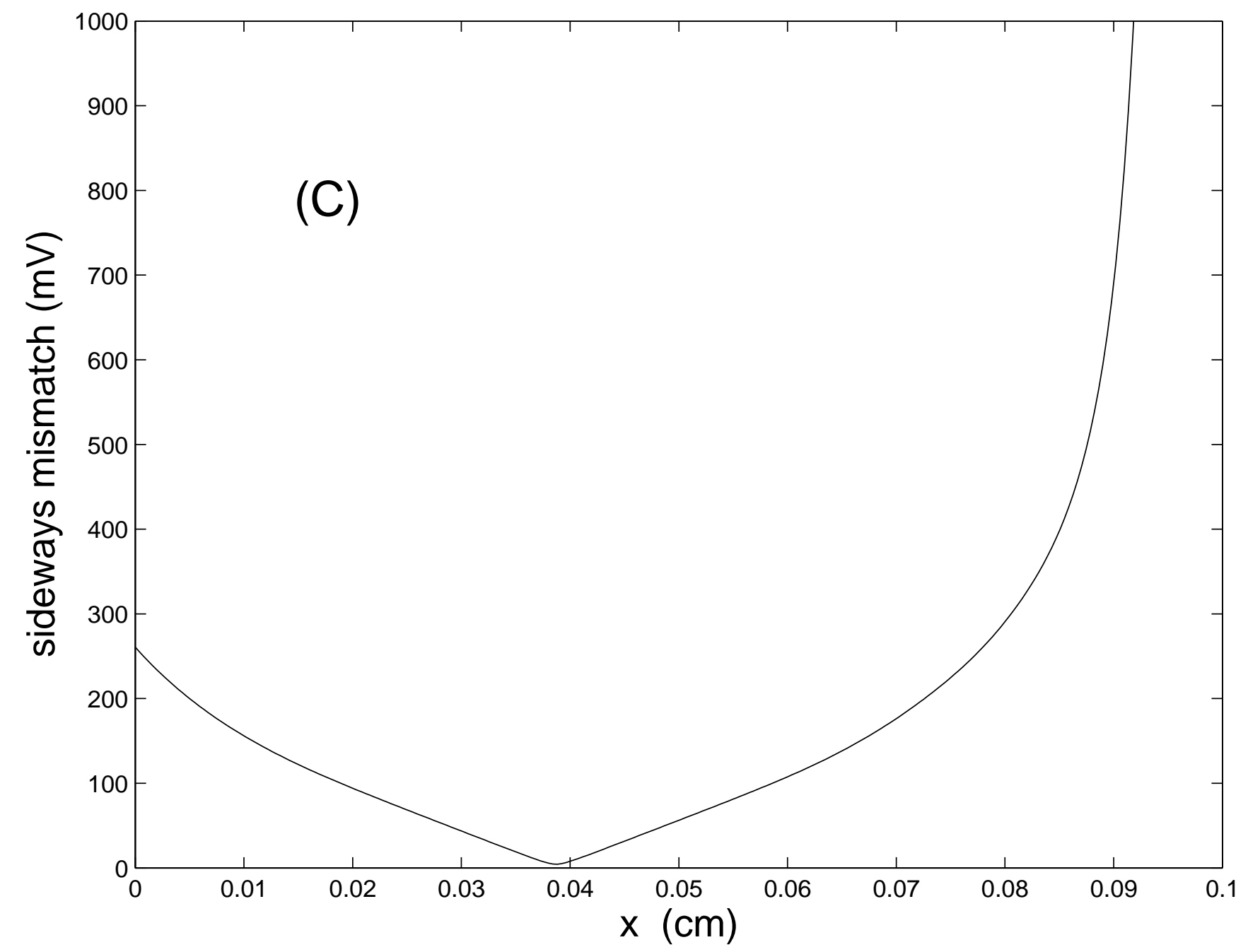


Figure $7(\mathrm{D})$

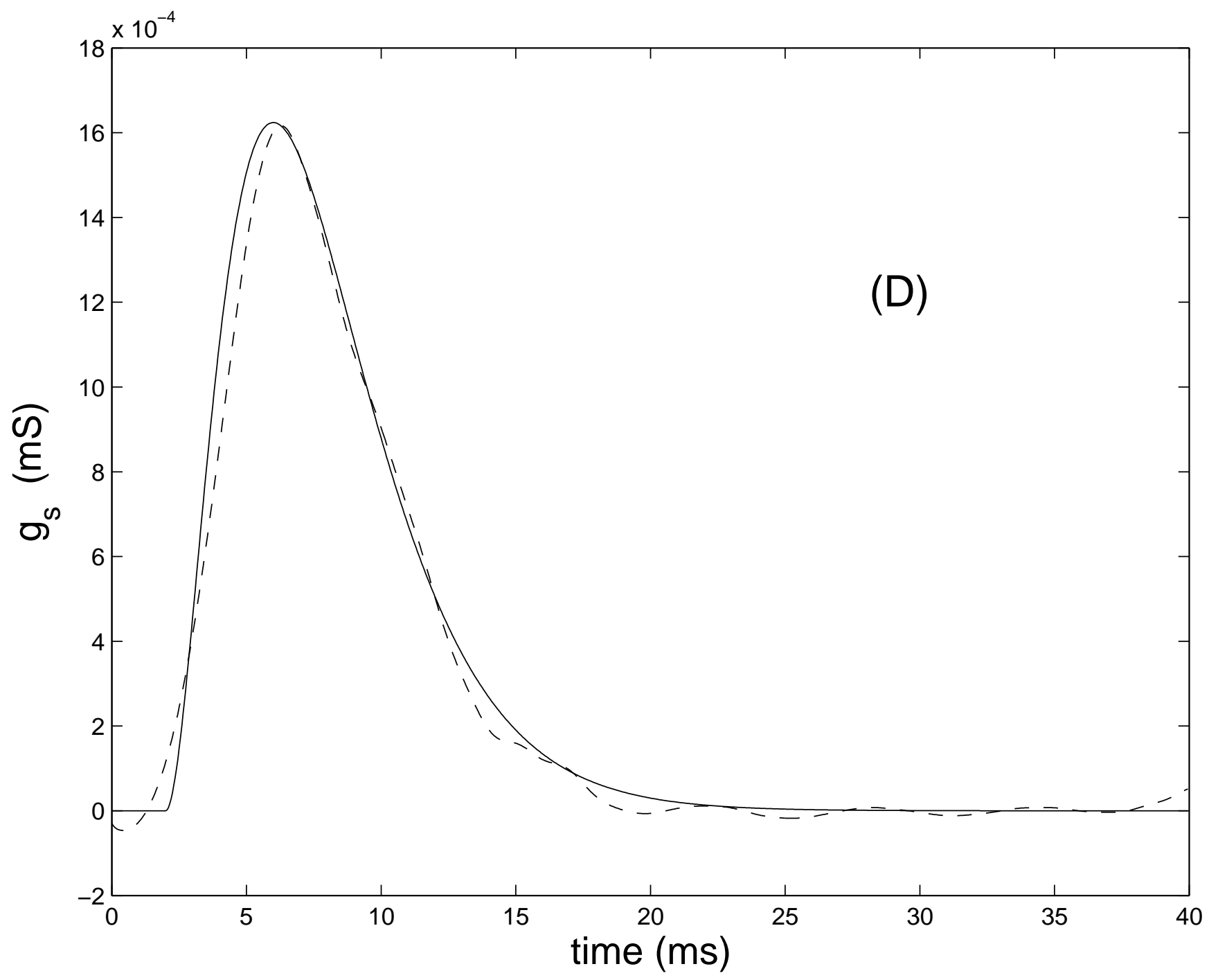


Figure 8(A)

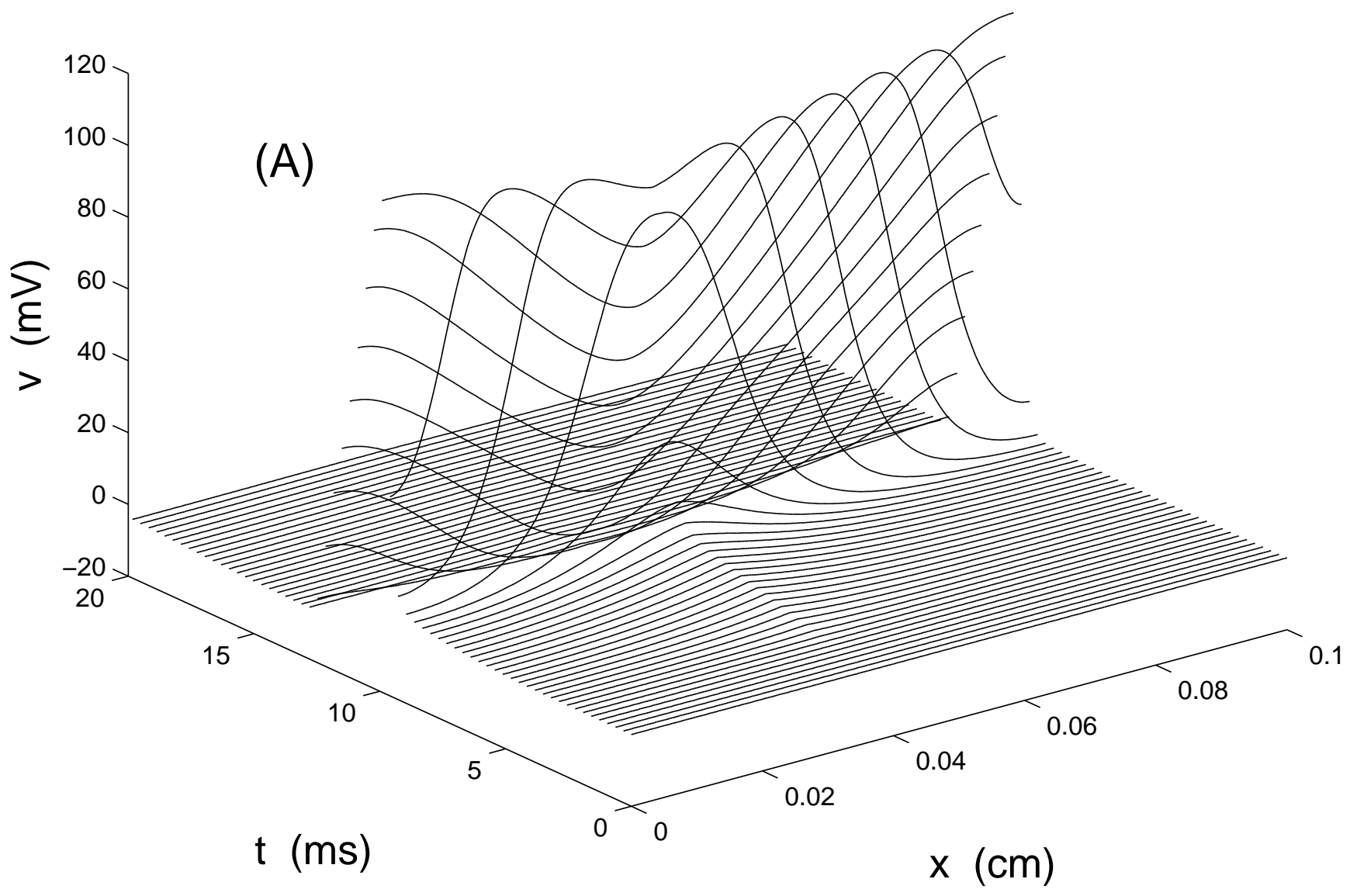


Figure 8(B)

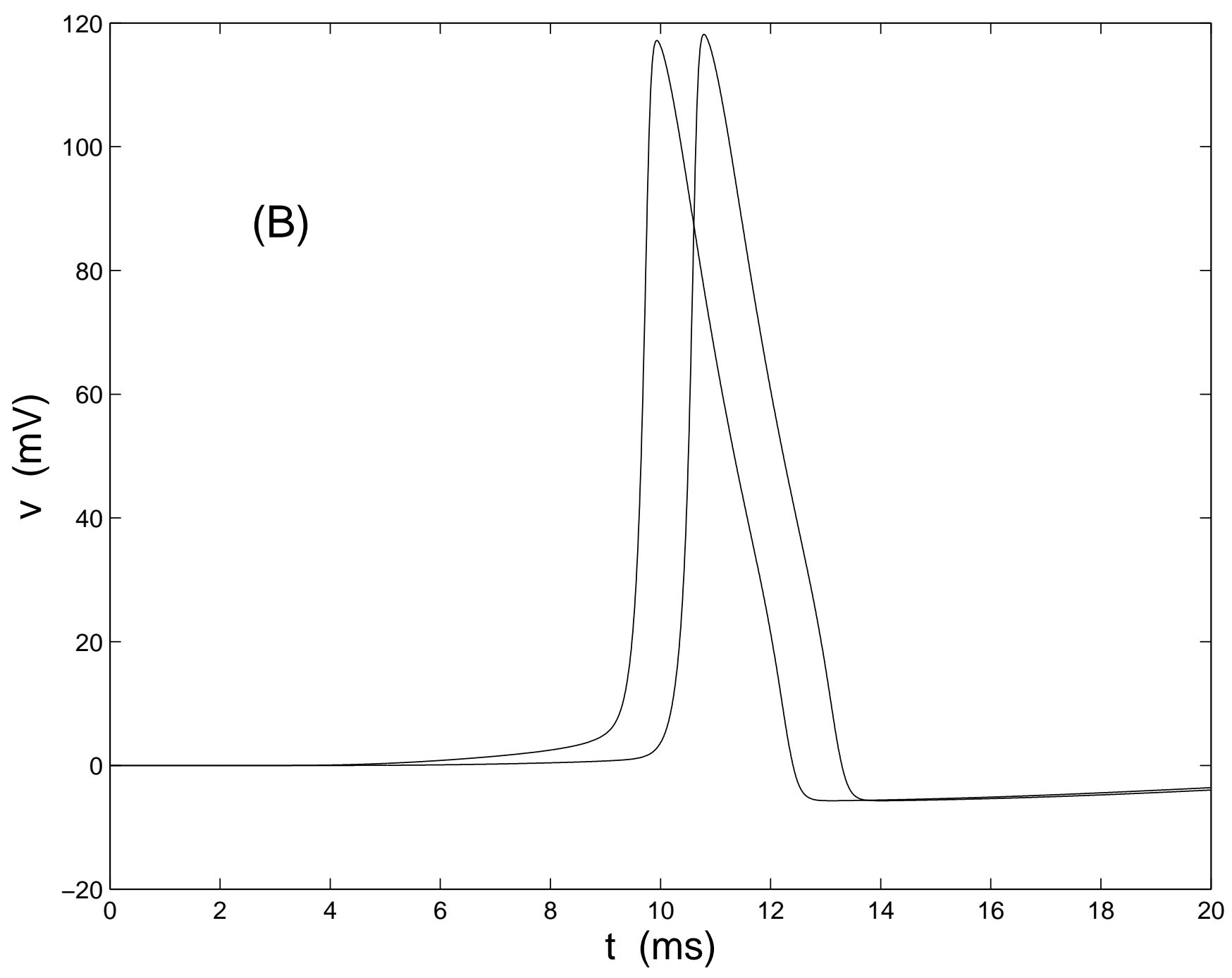

